\title{
8. MINERALOGY OF HYDROTHERMALLY ALTERED SEDIMENTS AND IGNEOUS ROCKS AT SITES 856-858, MIDDLE VALLEY, JUAN DE FUCA RIDGE, LEG 139¹
}

\author{
Viktor Kurnosov, ${ }^{2}$ Ivar Murdmaa, ${ }^{3}$ Tamara Rosanova, ${ }^{3}$ Georgy Kashintzev, ${ }^{3}$ Vadim Eroshchev-Shak, ${ }^{2}$ and Sergey Krasnov ${ }^{4}$
}

\begin{abstract}
Mineralogical analyses of hydrothermally altered sediments and basalts recovered from Leg 139 boreholes in Middle Valley of the Juan de Fuca Ridge reveal an increasing intensity of alteration, from the least affected Holes $856 \mathrm{~A}$ through $856 \mathrm{~B}$ and composite section at Site 857, to the most affected at Site 858, drilled within an active hydrothermal vent area. Three main hydrothermal zones are recognized in the sediment sequence at Sites 856,857 , and 858 . The lowermost zone, Zone III, is strongly hydrothermally altered and contains pyrite, chlorite, quartz, epidote, wairakite, and analcime. The mineral assemblages both in strongly altered sediments and in interbedded basaltic sills indicate alteration under the greenschist metamorphic facies. Zone II contains hydrothermal pyrite, chlorite, a corrensite-like mineral, anhydrite, and gypsum; quartz and carbonate are also present. The uppermost zone, Upper Zone I, is weakly altered and contains mainly clastic sediments and authigenic smectites.
\end{abstract}

\section{INTRODUCTION}

Leg 139 occupied four sites located in Middle Valley of the Northern Juan de Fuca Ridge (Fig. 1) to study processes and products associated with hydrothermal circulation in a sedimented spreading center. This article reports the results of mineralogical analyses of sediment samples from Holes 856A and 856B; Holes 857A, 857C, and 857D; Holes $858 \mathrm{~A}, 858 \mathrm{~B}, 858 \mathrm{C}$, and $858 \mathrm{D}$; and igneous rocks from Holes $856 \mathrm{~A}, 857 \mathrm{C}, 857 \mathrm{D}, 858 \mathrm{~F}$, and $858 \mathrm{G}$. The goal of the article is to discuss and interpret hydrothermal alteration of the sediments. The samples we received from Leg 139 contain low or negligible amounts of sulfides; consequently, this article does not elucidate questions about sulfide mineralogy.

\section{SITES AND SETTINGS}

Site 855 was located near the eastern boundary fault scarp. Mafic igneous rocks were encountered at 74 mbsf. Heat flow is low at Site 855. Unaltered hemipelagites and turbidites are considered to represent "background" sediment.

Site 856 was located near a small hill in the eastern part of Middle Valley, about $3 \mathrm{~km}$ west of the eastern boundary fault scarp and $4 \mathrm{~km}$ east of the present-day rift axis. Holes $856 \mathrm{~A}$ and $856 \mathrm{~B}$ were located at the center and on the southern edge of the hill. There is a smal active vent field roughly $200 \mathrm{~m}$ south of Site 856 . Hot (up to $265^{\circ} \mathrm{C}$ ) water is discharging there. Coring in Holes $856 \mathrm{~A}$ and $856 \mathrm{~B}$ recovered basalts from thick sills at $112 \mathrm{mbsf}$ and $120 \mathrm{mbsf}$, respectively. The basalts are overlain by undisturbed semi-indurated Pleistocene turbidites. Temperatures at the bottom of the holes are about $60^{\circ} \mathrm{C}$ in Hole $856 \mathrm{~A}$ and $160^{\circ} \mathrm{C}$ in Hole $856 \mathrm{~B}$.

Site 857 is located $1.6 \mathrm{~km}$ from the hydrothermal vent field at Site 858 and $5.2 \mathrm{~km}$ west of the eastern boundary fault scarp. The site is located on a major thermal anomaly, where the seafloor heat flow exceeds $0.8 \mathrm{Wm}^{-2}$. Hole $857 \mathrm{~A}$, located slightly west of the peak in heat flow, was drilled to $112 \mathrm{mbsf}$ in an area with a heat flow of about $0.7 \mathrm{Wm}^{-2}$. Hole $857 \mathrm{C}$ is located $180 \mathrm{~m}$ from Hole $857 \mathrm{~A}$ in an area of

${ }^{1}$ Mottl, M.J., Davis, E.E., Fisher, A.T., and Slack, J.F. (Eds.), 1994. Proc. ODP, Sci. Results, 139: College Station, TX (Ocean Drilling Program).

${ }^{2}$ Geological Institute, Pyzhevsky Per. 7, Moscow 109017, Russia

${ }^{3}$ Institute of Oceanology, Krasikov St. 23, Moscow 117218, Russia

4 “"Oceangeologia," Moika St. 120, St. Petersburg 190121, Russia. measured heat flow of about $0.8 \mathrm{Wm}^{-2}$. This hole was drilled to 567.7 mbsf, where a temperature greater than $250^{\circ} \mathrm{C}$ was estimated. Mafic sills interlayered with sediments were encountered between 471.1 and 567.7 mbsf. Hole 857D, a deep reentry hole, was drilled $50 \mathrm{~m}$ north of Hole $857 \mathrm{C}$ to a total depth of $936.2 \mathrm{mbsf}$. Mafic sills and interlayered sediments were cored between 581.1 and $936.2 \mathrm{mbsf}$.

Site 858 is located $1.6 \mathrm{~km}$ north of Site 857 at an active hydrothermal vent field where numerous vents with temperatures ranging from 255 to $276^{\circ} \mathrm{C}$ have been observed. Hole $858 \mathrm{~A}$ is located about $100 \mathrm{~m}$ west of the vent field area and about $150 \mathrm{~m}$ west of the nearest currently active vents. The calculated thermal gradient is $1.7^{\circ} \mathrm{C} \mathrm{m}^{-1}$ Hole $858 \mathrm{C}$ is located within the distal part of the vent field area, $70 \mathrm{~m}$ west of the nearest vent. The downhole temperature gradient is $3^{\circ} \mathrm{C}$ $\mathrm{m}^{-1}$. Hole $858 \mathrm{~B}$ is located $140 \mathrm{~m}$ east of Hole $858 \mathrm{C}$ and only a few meters away from a $276^{\circ} \mathrm{C}$ hydrothermal vent. A temperature of $197^{\circ} \mathrm{C}$ was measured at $19.5 \mathrm{mbsf}$. Hole $858 \mathrm{D}$ is located at the center of the vent field, about $70 \mathrm{~m}$ northeast of the nearest vent (at Hole $858 \mathrm{~B}$ ) where a temperature of $208^{\circ} \mathrm{C}$ was measured at $18.5 \mathrm{mbsf}$ Hole $858 \mathrm{~F}$ ( $258 \mathrm{mbsf}$ ) and deep reentry Hole $858 \mathrm{G}$ (432.6 mbsf) were drilled approximately in the center of the field.

\section{METHODS}

Sediments were studied in smear-slides and thin sections. X-ray diffraction (XRD) mineralogy was determined for bulk powder samples, for clay fraction (less than $0.001 \mathrm{~mm}$ ), and in rare samples for $0.001-0.01-\mathrm{mm}$ fractions. These fractions were precipitated from suspension and prepared as oriented specimens.

Secondary minerals from igneous rocks were studied in thin sections and analyzed by XRD by oriented specimens prepared from suspensions using distilled water and by grinding rock samples (1-2 $\mathrm{mm}$ ) in an agate mortar. Clay minerals were concentrated in the suspensions. A few samples were examined by microprobe.

A DRON-1.5 X-ray diffractometer with $\mathrm{CuK}_{\alpha}$ emission, $\mathrm{Ni}$-filter, and slot openings of $0.5,1,1,0.5 \mathrm{~mm}$ was used. Each sample was dried in air, treated with glycerine, and heated at $550^{\circ} \mathrm{C}$ for 1 to 2 hours. For quantitative counting of clay minerals the method of Sudo et al. (1969) was used. They proposed the following mean intensity coefficients: (Montmorillonite) $:$ (Hydromica) $:($ Chlorite) $=3.6: 1: 1$.

The coarse fraction $(0.1-0.05 \mathrm{~mm})$ was separated by wet sieving and studied using oil immersion. Heavy and light fractions were separated in bromoform $\left(2.9 \mathrm{~g} / \mathrm{cm}^{3}\right)$. Most samples were examined by scanning electron microscope (SEM). 


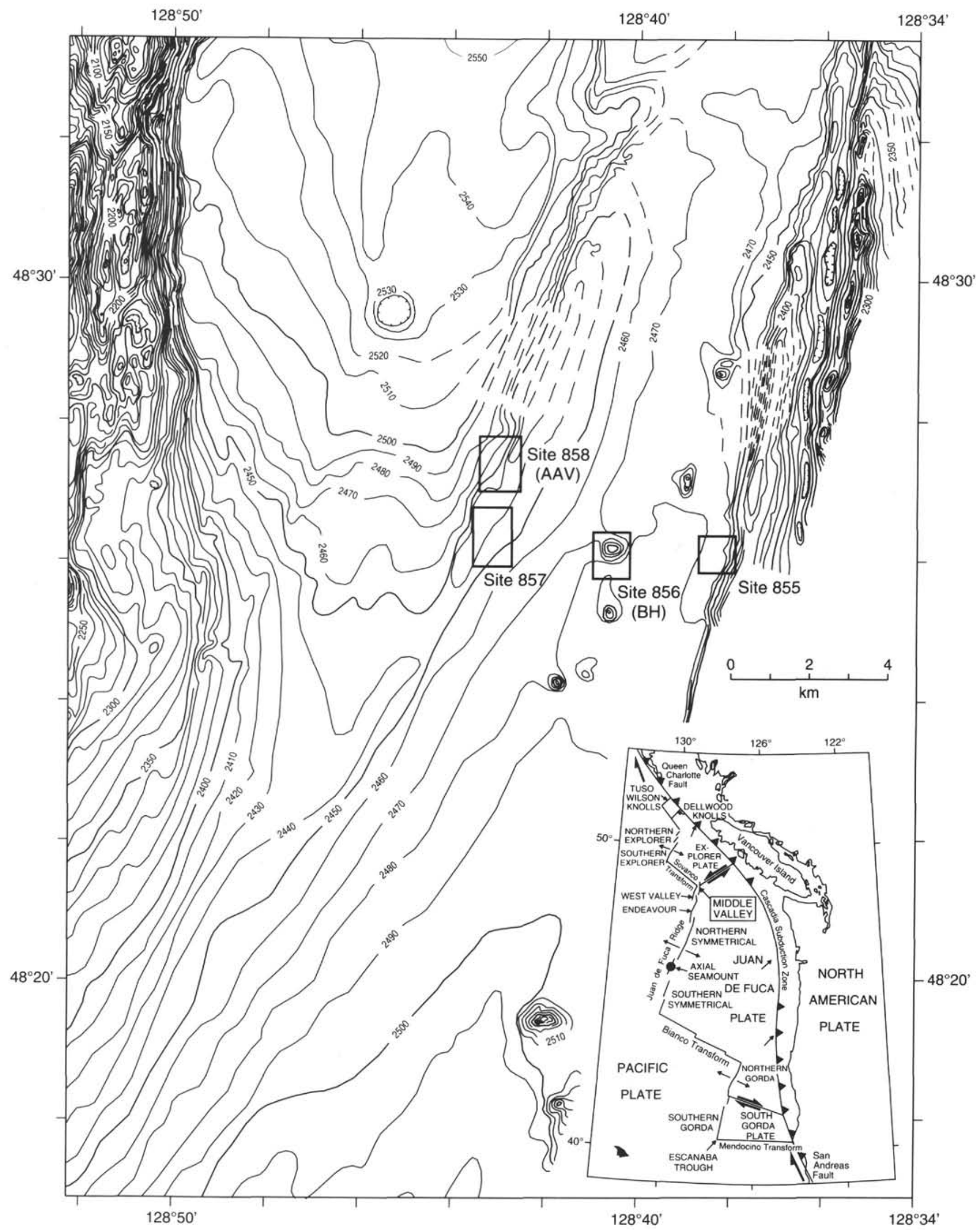

Figure 1. Site map showing locations of Sites $855-858$ in Middle Valley. 


\section{MINERALS IN SEDIMENTS}

\section{Minerals}

Chlorites, hydromica, smectites, and corrensite-like minerals were determined in sediments from Leg 139 by routine XRD methods. Fe chlorites are widely distributed in the sediments. They have low intensity (001) reflections at $14 \AA$ and high-intensity (002) reflections at 7 $\AA ̊$ (Fig. 2, Sample 139-857C-65R-1, 12-14 cm). Hydromica commonly has a (001) reflection at 9.94 to $9.98 \AA$ (Fig. 2, Sample 139$857 \mathrm{C}-27 \mathrm{R}-2,81-86 \mathrm{~cm}$ ), which indicates that hydromica contains blocks with $\mathrm{K}$ and $\mathrm{Na}$ components. The hydromica is dioctahedral, with a (002) reflection near $4.97 \AA$. Smectites exhibit the (001) reflection at $17.6-17.8 \AA$ in glycerine-saturated specimens (Fig. 2, Sample 139-857C-11R-1, 103-105 cm). A corrensite-like mineral was found in sediments from Hole 858B. Typical reflections are shown in Figure 3 (Sample 139-858B-2H-4, 68-70 cm).

The following nonclay minerals were detected by X-ray diffraction, thin section, and immersion analysis: quartz and feldspar, amphibole, biotite, muscovite, pyrite, iron oxides, anhydrite, gypsum, barite, carbonates, epidote, wairakite, analcime, clinoptilolite (?), rutile, anatase, sphene, zircon, garnet, apatite, zoisite and trace minerals (chalcedony, opal A, volcanic glass, talc [?], tremolite and/or actinolite, black opaques, olivine, leucoxene).

\section{Origin of Minerals}

Unaltered hemipelagites and turbidites recovered at Site 855 are considered to represent "background." The Shipboard Scientific Party (Davis, Mottl, Fisher, et al., 1992) determined that "background" sediments consist predominantly of quartz and plagioclase feldspar with subordinate amounts of amphibole and mica. The clay-size fraction is predominantly composed of chlorite and hydromica. This mineral assemblage reflects a mainly terrigenous origin.

Sediments recovered at Sites 856,857 , and 858 often contain altered or recrystallized detrital minerals and newly-formed quartz, possibly feldspar, amphibole, as well as a group of evidently hydrothermal minerals (including anhydrite, wairakite, analcime, corrensite-like mineral, often chlorite).

\section{Clay Minerals}

Hydromica has both a detrital and an authigenic origin. Authigenic hydromica as radial aggregates was found by SEM and confirmed by microprobe measurement of potassium (Plate 1, Figs. 1-3).

Chlorite is also both detrital and authigenic. Often the two types are intermixed and difficult to distinguish. Authigenic chlorite is common as cement in siltstone, where it shows an apparently authigenic texture. The claystone is predominantly composed of chlorite, which is also apparently authigenic, based on intergrowth structures including spheroidal cabbage-like aggregates and stacks of parallel or diverging sheets. The crystalline structure of chlorite is well demonstrated by SEM images (Plate 1, Figs. 4-7). Chlorite is authigenic in origin where the clay fraction is composed of pure chlorite. Small pores and amygdules are in some cases encrusted or infilled by newly-formed chlorite. Chlorite also replaces feldspar and mica.

The mixed-layer corrensite-like mineral is authigenic (hydrothermal) in origin. The structure of this mineral is shown in Plate 1, Figure 8 .

Almost all the smectites are of authigenic origin.

\section{Quartz and Feldspar}

Quartz and feldspar in the coarse fraction of sediments are mainly terrigenous clastic minerals. Thin sections show recrystallization (from weak to full) of the detrital quartz cementing silty sandstone and clayey siltstone. The mosaic (anhedral) authigenic quartz occurs both in veinlets and as recrystallized sandy laminae. Hydrothermal

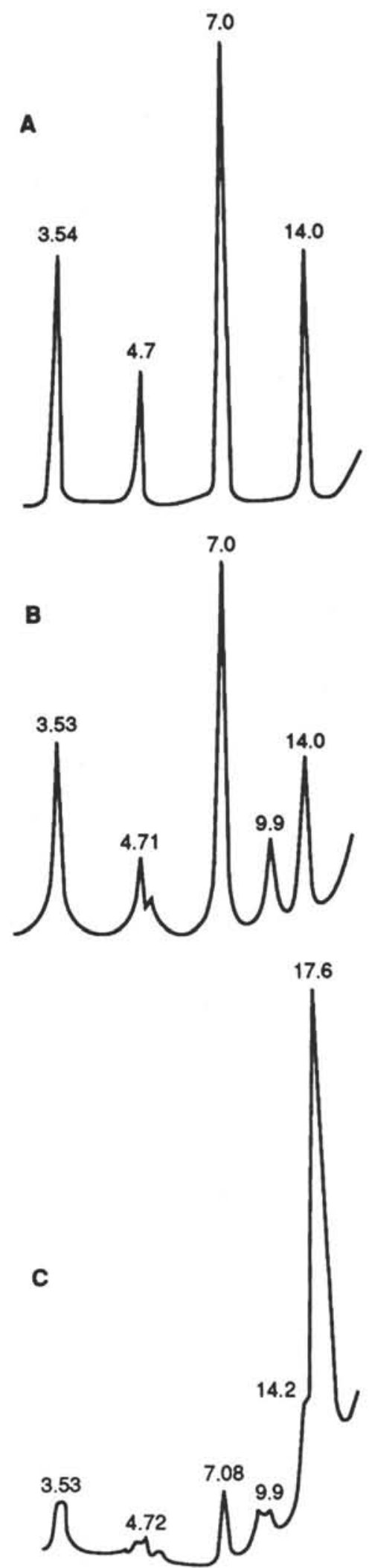

Figure 2. X-ray diffraction patterns of less than $0.001 \mathrm{~mm}$ fraction from sediments. A. Fe chlorite (Sample 139-857C-65R-1, 12-14 cm, air-dried). B. Fe chlorite and hydromica (Sample 139-857C-27R-2, 81-86 cm, air-dried). C. Smectite, hydromica, and chlorite (Sample 139-857C-11R-1, 103-105 cm, glycerine-saturated). 


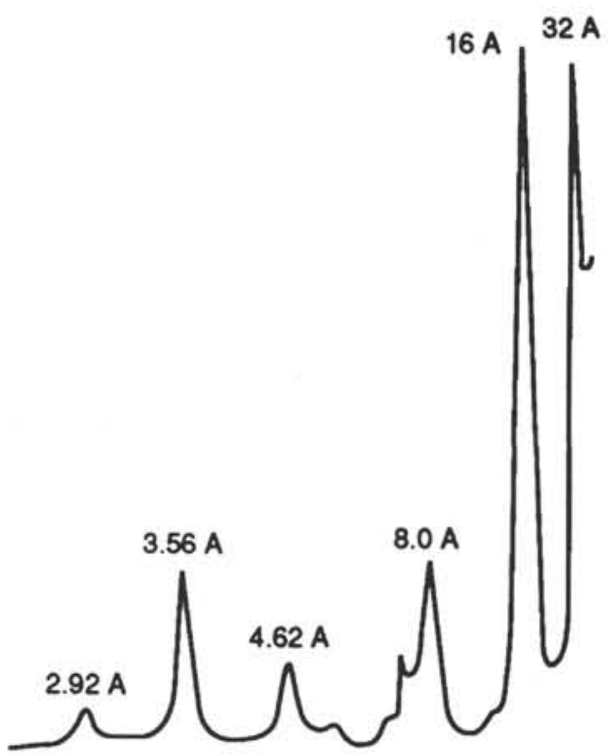

Figure 3. X-ray diffraction pattern of corrensite-like mineral and chlorite (less than $0.001 \mathrm{~mm}$ fraction, Sample 139-858B-2H-4, 68-70 cm, glycerine-saturated) from sediments.

euhedral quartz crystals have grown in fissures and cavity walls. Detrital feldspar is replaced by chlorite, epidote, and sericite. Some feldspar in trace amounts may be authigenic in quartz-chlorite metamorphic rocks.

\section{Amphibole}

Detrital amphibole totally disappears from the sediments with depth in all of the holes. A fibrous amphibole-like mineral was observed by SEM (Plate 2, Fig. 1) infilling pores and replacing foraminiferal tests. Fibrous amphibole of similar appearance has been described previously in a low-temperature hydrothermally altered sediment sample dredged from the Hess Deep (Rosanova et al., 1978).

\section{Mica}

Detrital mica (both biotite and muscovite) is affected by chloritization.

\section{Pyrite}

Disseminated authigenic pyrite occurs as small cubic crystals, spherulites (framboids), and irregular particles, which may replace organic fragments in all clay and silty clay samples. Both diagenetic and hydrothermal origins are possible for this type of pyrite; however, the concentration of disseminated pyrite in some thin sections seems too high for common diagenetic processes. The diagenetic pyritization may be accelerated by hydrothermal activity. Pyrite of apparently hydrothermal origin occurs as crystalline aggregates in pore spaces, as small veins, and as incrustations around anhydrite crystals. Early diagenetic or hydrothermal pyrite also occurs as burrow fillings and replaces foraminifers and diatoms.

Large rectangular (crystal-like) or irregular pyrite segregations composed of fine-crystalline pyrite aggregates occur both in claystone and in siltstone layers. Their crystal-like rectangular shape may be inherited from another mineral (anhydrite ?) that was replaced by pyrite, forming pseudomorphs.

\section{Anhydrite and Gypsum}

Anhydrite and gypsum occur as crystalline aggregates infilling small fractures and caverns and as poikilitic inclusions, with sediment incorporated into the skeletal crystals. Textural intercalations in the sulfates indicate replacement of anhydrite by gypsum. Large segregations (veins?) of euhedral elongated anhydrite crystals occur as radiated or parallel intergrowths, with pyrite infilling interstitial space between the crystals. Authigenic forms of sulfates are shown in Plate 2 , Figures 2,3 , and 4 .

\section{Carbonates}

Carbonate minerals include biogenic foraminiferal tests and fragments, "unidentified" fragments, and crystalline calcite (which could be recrystallization products of biogenic carbonate), coarse-crystalline (sparry) calcite cement, detrital grains, diagenetic calcite concretions, microconcretions and burrow-fills. Authigenic carbonate fills pore space. Calcite veins cut brecciated sediments.

\section{Epidote}

Epidote occurs both as detrital grains and as authigenic crystals. The latter occur in cement as euhedral crystals (Plate 2, Figs. 5 and 6) and as aggregates of parallel or radial crystals and rims around clastic grains. In some cases epidote-clinozoisite seems to replace plagioclase. It also corrodes crystals of pyrite. The epidote forms stringers and veinlets.

\section{Zeolites}

Wairakite/analcime veinlets are composed of euhedral crystals (Plate 2, Figs. 8 and 9). Unidentified zeolite (clinoptilolite ?) and rare analcime were detected in the coarse light fraction. They are either hydrothermal or diagenetic in origin.

\section{Rutile and Anatase}

The titanium-bearing minerals rutile and anatase appear to be of authigenic origin. They fill interstitial space together with opaque minerals (pyrite) and incrust small pores in some cases. We found also small segregations of a finely crystalline titanium mineral (anatase ?) in Hole 858D.

\section{Other Minerals}

Barite is possibly authigenic. Garnet, zircon, sphene, and apatite are likely to be authigenic in part. Zoisite or clinozoisite replaces plagioclase.

\section{SECONDARY MINERALS OF BASALTS}

Secondary minerals in sills (chlorite, corrensite-like mineral, epidote, uralite, prehnite, quartz, albite, chalcedony, zeolite, leucoxene, sphene, and sulfides) were formed by hydrothermal alteration.

Chlorites with different $\mathrm{Fe} / \mathrm{Mg}$ ratios are the principal secondary minerals in the igneous rocks recovered during Leg 139. Fe-rich chlorites have low-intensity (001) reflections at $14 \AA$ and high-intensity (002) reflections at $7 \AA$ (Fig. 4, Sample 139-857C-63R-1, 50-52 $\mathrm{cm}) . \mathrm{Fe}-\mathrm{Mg}$ chlorites with $\mathrm{Fe}$ slightly predominating over $\mathrm{Mg}$ have an (001) reflection that is less intense than the (002) reflection (Fig. 4, Sample 139-857C-59R-1, 20-22 cm). Mg-Fe chlorites have an (001) reflection higher than (002) (Fig. 4, Sample 857C-61R-2, 8-10 $\mathrm{cm}$ ). Chlorites may contain hydrated interlayers (rarely up to $10 \%$ ). In this case, the (001) reflection moves to $14.5-14.7 \AA$ in glycerinesaturated specimens.

Corrensite-like minerals are found together with chlorites. Typical reflections of the corrensite-like minerals are shown in Figure 5 (Sample 139-857C-60R-1, 130-132 cm).

\section{DOWNHOLE DISTRIBUTION OF MINERALS AND ZONATION}

$\mathrm{X}$-ray diffraction results are listed in Tables 1 and 2, immersion results in Table 3, and petrographical and X-ray diffraction results for 
7.0
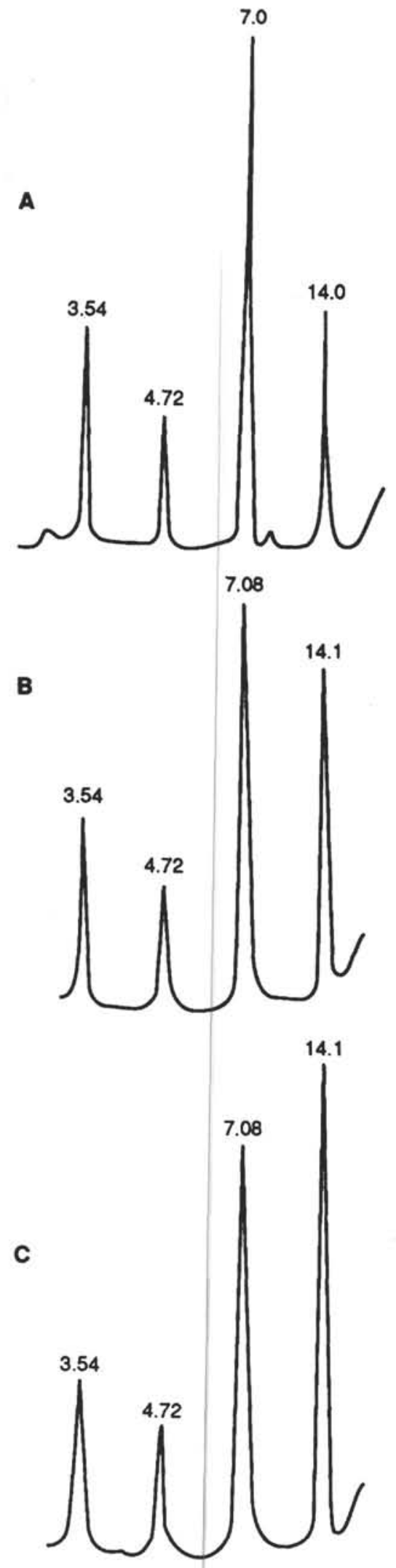

Figure 4. X-ray diffraction patterns of chlorites from igneous rocks (specimens are air-dried). A. Fe chlorite, Sample 139-857C-63R-1, 50-52 cm; B. Mg-Fe chlorite, Sample 139-857C-59R-1, 20-22 cm; C. Fe-Mg chlorite, Sample 139-857C-61R-1, 8-10 cm.

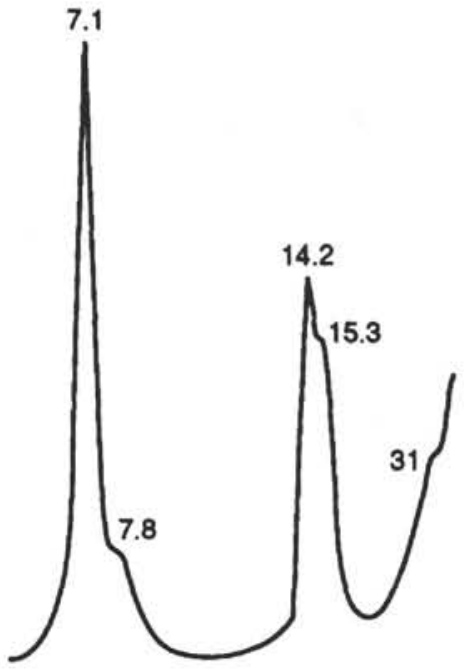

Figure 5. X-ray diffraction pattern of $\mathrm{Mg}$-Fe chlorite and corrensite-like mineral (Sample 139-857C-60R-1, 130-132 cm, saturated by glycerine).

altered basalts in Table 4. The mineralogical data show that all sections at Sites 856,857 , and 858 are divided into three main zones with some variations in intensity of alteration and in complexity of the mineralogical assemblages.

\section{Site 856}

The Quaternary sediments recovered at Site 856 were divided by shipboard scientists into two lithostratigraphic units. Lithologic Unit I is represented by massive silty clay, $2.32 \mathrm{~m}$ thick. Unit II is characterized by alternating hemipelagic and turbiditic sediments that are variously altered. Subunit IIA is weakly altered, Subunit IIB was recognized as a slump(?) deposit and is present in Hole 856B only, Subunit IIC is moderately indurated and altered, and Subunit IID is moderately to well indurated and contains abundant sulfide minerals.

\section{Hole $856 A$}

Alteration Zone I coincides with lithologic Unit I (0.00-2.32 mbsf). Zone II coincides with lithologic Subunits IIA and IIC ( 2.32 to $113.22 \mathrm{mbsf}$ ).

In Zone II the chlorite content gradually increases downhole, from $56 \%$ to $81 \%$ of the total fraction of clay minerals, while hydromica decreases. Smectite is rare (Table 1, Fig. 6). Anhydrite and gypsum are present in trace amounts throughout the section. Quartz, feldspar, chlorite, and mica are the dominant detrital phases. Amphibole of possibly authigenic origin was detected by XRD (Table 2 ) in the uppermost part of the section (6.3 mbsf).

The heavy-mineral fraction in Hole $856 \mathrm{~A}$ is dominated by pyrite (Table 3 ). The pyrite crystals in many samples are oxidized, so that $\mathrm{Fe}$-oxide particles constitute up to $80 \%$ of the heavy fraction. Epidote occurs both as detrital grains and as crystal aggregates. Siltstone samples show weak recrystallization of quartz.

Secondary minerals were studied in a single sample of basalt. The basalt is weakly altered (Table 4) in a manner similar to the overlying sediments. Olivine is replaced by iddingsite and talc (Table 5). Plagioclase is replaced by prehnite and chlorite.

Mineralogical data show that the terrigenous "background" sediments in Hole 856A are affected by some hydrothermal alteration. Indicators of this weak alteration are traces of authigenic anhydrite, gypsum, pyrite, and amphibole; increase of chlorite content and dissolution of biogenic carbonate downward; and weak recrystallization of quartz below $70 \mathrm{mbsf}$. 


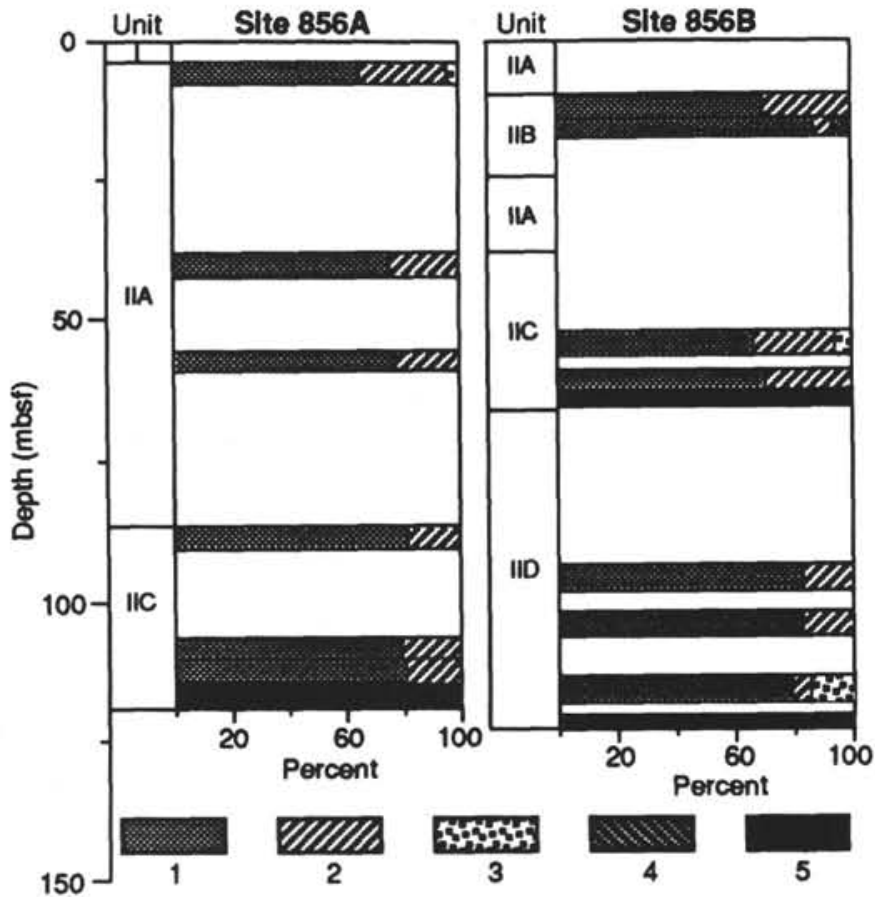

Figure 6. Variations in clay mineral composition of the clay fraction, Site 856. 1 = chlorite; 2 = mica; 3 = smectite; 4 = corrensite-like mineral; $5=$ basalt.

\section{Hole $856 B$}

The style of alteration in Hole $856 \mathrm{~B}$ is the same as that in Hole $856 \mathrm{~A}$, but more intense.

Zone II ( 0.00 to $65.44 \mathrm{mbsf})$ coincides with lithologic Subunits IIA, IIB, and IIC and includes among the authigenic minerals mainly quartz, pyrite, chlorite, and anhydrite. Hole $856 \mathrm{~B}$ contains a larger amount of pyrite and anhydrite than Hole 856A. Feldspar decreases with depth (Table 2). Hydrothermal dissolution of biogenic carbonates occurs at a much shallower depth in Hole 856B (below Core 139-856B-1H, Section 1) than in Hole 856A (below $55 \mathrm{mbsf}$ ). In one thin section from Hole 856B (45.2 mbsf), silty sandstone is extensively carbonatized. The carbonate is developed as patchy cement.

The sandstone contains moderately abundant titanium-bearing minerals (rutile and anatase) that exhibit an authigenic appearance. They fill interstitial space together with pyrite. Other minerals occasionally present in trace quantities are garnet, zircon, sphene, apatite, and barite, with rare olivine. An unidentified zeolite (clinoptilolite?) is present in the light fraction (Table 3), which occurs throughout the section in both holes.

The slump(?) sediments (Subunit IIB) contain abundant chlorite with traces of the corrensite-like mineral. This mineral assemblage reflects more intense alteration than that in Subunit IIA.

Zone III (70.80 to 120.51 mbsf) coincides with lithologic Subunit IID and includes among the authigenic minerals mainly pyrite, chlorite, and quartz. Disseminated pyrite and possibly other sulfides are abundant (Table 3). Pyrite occurs as large rectangular pseudomorphs. Authigenic quartz occurs as mosaic cement and fissure and cavity filling. Feldspar is rare or absent (Table 2) and has been replaced by quartz and Fe chlorite. Authigenic chlorite occurs in cement (Table 1, Fig. 6). Authigenic hydromica occurs as radial aggregates. Anhydrite is absent.

\section{Site $\mathbf{8 5 7}$}

Lithologic Unit I is a Holocene to upper Pleistocene silty clay (hemipelagic sediments and turbidites). The degree of induration and hydrothermal alteration was used by shipboard scientists to subdivide the Pleistocene turbidites (Unit II) into three Subunits: IIA, IIB and IIC. Sediments of Subunit IIA exhibit little induration. Sediments of
Subunit IIB are moderately to strongly indurated. Sediments of Subunit IIC are strongly indurated and hydrothermally altered, and are interbedded with igneous rocks. Geochemically the composite sequence drilled at Site 857 was divided into two zones with a boundary somewhere near $400-450$ mbsf.

Mineralogical data distinguish three zones of hydrothermal alteration in sediments recovered at Site 857: Uppermost Zone I (0 to 145-176 mbsf) with weakly altered sediments, Zone II (145-176 to $400-450$ mbsf) with moderately altered sediments, and lowermost Zone III ( $400-450$ to $926 \mathrm{mbsf})$ with strongly altered sediments interbedded with sills.

Alteration Zone I coincides with lithologic Unit I, Subunit IIA, and the uppermost part of Subunit IIB. Sediments from this zone are characterized by a high content of smectite (Table 1, Fig. 7). Detrital minerals include quartz and feldspar (Table 2). Amphibole (of possibly authigenic origin) was found by XRD in the uppermost sample (69 mbsf). Quartz and feldspar predominate in the light fraction, with minor mica and a high content of biogenic carbonate (Table 3). The feldspars are represented mainly by sodium plagioclase and potassium feldspar with minor andesine-labradorite.

The carbonate minerals, including biogenic foraminiferal tests and fragments, "unidentified" fragments, and crystalline calcite, which could be a recrystallization product of the biogenic carbonate, are present at high percentages (up to 66\%) to about 125 mbsf (Table 3). Below this depth the carbonate content decreases sharply, indicating a transition from the carbonate recrystallization and cementation zone to the carbonate dissolution zone.

The heavy fraction (Table 3) contains pyrite and iron oxides resulting from pyrite oxidation. Garnet, zircon, sphene, anatase, apatite, pyroxenes, barite, anhydrite, and "black opaques" (ilmenite?) occur occasionally as traces.

Zone II coincides with lithologic Subunit IIB and contains among authigenic minerals mainly quartz, pyrite, chlorite, and anhydrite.

This zone does not show any significant changes in the major elements relative to "background" values (shipboard data). The major constituents are quartz and feldspar (Tables 2 and 3). Their relative proportions are almost constant, with quartz predominating over feldspar.

The clay fraction is represented by Fe chlorite and less abundant hydromica; smectites are absent (Table 1, Fig. 7). Authigenic carbonate and anhydrite infill pore space in sandy layers. An unidentified zeolite occurs occasionally (Table 3 ). Calcite and anhydrite were not identified by XRD (Table 2). We observed large rectangular or irregular pyrite segregations composed of finely crystalline pyrite aggregates, and mosaic quartz recrystallization.

Thin section analyses from 155 to 414 mbsf indicate a general downhole increase in hydrothermal effects. More altered sandy layers or laminae are interbedded with less altered claystones. The hydrothermal alteration is manifested by two main processes: pyrite precipitation and quartz recrystallization. Chlorite with authigenic textures is visible in the lower part of the zone, especially in sandstonesiltstone interbeds, where chlorite cements the detrital minerals.

Zone III coincides with lithologic Subunit IIC and contains among authigenic minerals mainly pyrite, chlorite, epidote, and quartz.

In this zone the clay fraction is totally composed of Fe chlorite (Fig. 7). Thin sections from 529 mbsf to the bottom of Hole 857D at 927 mbsf show strong quartz cementation and a downhole increase in authigenic (hydrothermal) epidote-clinozoisite minerals. The quartzification is apparent even in fine-grained clayey siltstone (529.22 mbsf), where mosaic quartz occurs as small lenses within a siltstone matrix containing very fine-grained epidote. In bulk samples epidote is abundant (Table 2).

Epidotization becomes even more extensive downhole beginning at $829.7 \mathrm{mbsf}$. The sediment contains coarse-grained cubic crystals of pyrite, rimmed by epidote. Epidote forms stringers and veinlets with sulfides, in laminated silt and clayey silt. Strong quartz cementation is accompanied by possibly authigenic mica. In Sample 139-857D-28R$1,30-32 \mathrm{~cm}$ ( $839.2 \mathrm{mbsf})$ a mineral was detected by XRD that may be 
Table 1. Results of X-ray diffraction of clay minerals in sediments.

\begin{tabular}{|c|c|c|c|c|c|c|c|}
\hline $\begin{array}{l}\text { Core, section, } \\
\text { interval }(\mathrm{cm})\end{array}$ & $\begin{array}{l}\text { Depth } \\
\text { (mbsf) }\end{array}$ & Fraction & Unit & $\begin{array}{c}\text { Chlorite } \\
(\%)\end{array}$ & $\begin{array}{c}\text { Hydromica } \\
\text { (\%) }\end{array}$ & $\begin{array}{c}\text { Smectite } \\
(\%)\end{array}$ & $\begin{array}{c}\text { Corrensite } \\
(\%)\end{array}$ \\
\hline $\begin{array}{l}139-856 \mathrm{~A}- \\
2 \mathrm{H}-3,67-69 \\
5 \mathrm{H}-7,26-30 \\
7 \mathrm{H}-4,133-137 \\
11 \mathrm{X}-1,77-79 \\
13 \mathrm{X}-1,108-111 \\
13 \mathrm{X}-4,94-98\end{array}$ & $\begin{array}{r}6.37 \\
39.34 \\
56.03 \\
86.97 \\
106.38 \\
110.74\end{array}$ & $\begin{array}{l}3 \\
3 \\
3 \\
3 \\
3 \\
3 \\
3\end{array}$ & IIC & $\begin{array}{l}56 \\
74 \\
77 \\
82 \\
79 \\
81\end{array}$ & $\begin{array}{l}44 \\
26 \\
23 \\
18 \\
21 \\
19\end{array}$ & tr & \\
\hline $\begin{array}{l}139-856 \mathrm{~B}- \\
3 \mathrm{H}-1,108-110 \\
3 \mathrm{H}-2,58-62 \\
3 \mathrm{H}-2,58-62 \\
3 \mathrm{H}-3,92-96 \\
3 \mathrm{H}-3,92-96 \\
3 \mathrm{H}-4,62-66 \\
3 \mathrm{H}-4,62-66 \\
3 \mathrm{H}-4,62-66 \\
7 \mathrm{H}-4,46-49 \\
7 \mathrm{H}-4,46-49 \\
8 \mathrm{H}-2,74-76 \\
13 \mathrm{X}-4,84-88 \\
14 \mathrm{X}-2,97-100 \\
15 \mathrm{X}-4,73-75\end{array}$ & $\begin{array}{r}12.38 \\
13.38 \\
13.38 \\
15.22 \\
15.22 \\
16.42 \\
16.42 \\
16.42 \\
54.26 \\
54.26 \\
61.04 \\
96.44 \\
103.27 \\
115.63\end{array}$ & $\begin{array}{l}1 \\
1 \\
3 \\
1 \\
3 \\
1 \\
2 \\
3 \\
1 \\
3 \\
1 \\
3 \\
3 \\
1\end{array}$ & IID & $\begin{array}{l}70 \\
82 \\
71 \\
93 \\
84 \\
94 \\
96 \\
90 \\
67 \\
65 \\
72 \\
86 \\
83 \\
83\end{array}$ & $\begin{array}{r}30 \\
18 \\
29 \\
7 \\
16 \\
6 \\
4 \\
10 \\
33 \\
35 \\
28 \\
14 \\
17 \\
4\end{array}$ & 13 & $\begin{array}{l}\mathrm{tr} \\
\mathrm{tr}\end{array}$ \\
\hline $\begin{array}{l}139-857 \mathrm{~A}- \\
2 \mathrm{H}-2,8-10 \\
2 \mathrm{H}-2,8-10 \\
6 \mathrm{H}-3,40-42\end{array}$ & $\begin{array}{l}12.98 \\
12.98 \\
44.30\end{array}$ & $\begin{array}{l}1 \\
2 \\
1\end{array}$ & $\begin{array}{c}\text { I } \\
\text { IIA }\end{array}$ & $\begin{array}{l}31 \\
26 \\
29\end{array}$ & $\begin{array}{l}17 \\
17 \\
20\end{array}$ & $\begin{array}{l}52 \\
57 \\
51\end{array}$ & \\
\hline $\begin{array}{l}139-857 \mathrm{C}- \\
\text { 2R-2, 24-25 } \\
\text { 3R-2, 74-76 } \\
10 \mathrm{R}-1,72-74 \\
11 \mathrm{R}-1,103-105 \\
12 \mathrm{R}-2,47-50 \\
15 \mathrm{R}-3,74-76 \\
17 \mathrm{R}-2,112-116 \\
24 \mathrm{R}-1,44-48 \\
27 \mathrm{R}-2,81-86 \\
31 \mathrm{R}-2,7-9 \\
36 \mathrm{R}-2,139-143 \\
36 \mathrm{R}-2,139-143 \\
41 \mathrm{R}-3,48-50 \\
43 \mathrm{R}-2,20-23 \\
43 \mathrm{R}-2,20-23 \\
51 \mathrm{R}-2,75-78 \\
51 \mathrm{R}-2,75-78 \\
65 \mathrm{R}-1,12-14 \\
67 \mathrm{R}-1,28-30\end{array}$ & $\begin{array}{r}58.24 \\
68.74 \\
124.82 \\
134.83 \\
145.47 \\
176.24 \\
194.52 \\
259.64 \\
286.41 \\
324.27 \\
348.99 \\
348.99 \\
378.38 \\
386.40 \\
386.40 \\
425.65 \\
425.65 \\
529.22 \\
548.68\end{array}$ & $\begin{array}{l}1 \\
3 \\
1 \\
1 \\
1 \\
3 \\
1 \\
1 \\
1 \\
1 \\
1 \\
3 \\
1 \\
1 \\
3 \\
3 \\
1 \\
3 \\
1 \\
3 \\
3 \\
1 \\
1\end{array}$ & IIA & $\begin{array}{r}34 \\
50 \\
16 \\
8 \\
52 \\
72 \\
75 \\
82 \\
80 \\
77 \\
81 \\
81 \\
77 \\
79 \\
76 \\
82 \\
81 \\
100 \\
100\end{array}$ & $\begin{array}{r}17 \\
19 \\
13 \\
4 \\
16 \\
28 \\
25 \\
18 \\
20 \\
23 \\
19 \\
19 \\
23 \\
21 \\
24 \\
18 \\
19\end{array}$ & $\begin{array}{l}49 \\
31 \\
71 \\
88 \\
32\end{array}$ & \\
\hline $\begin{array}{l}139-857 \mathrm{D}- \\
11 \mathrm{R}-1,49-51 \\
16 \mathrm{R}-1,36-38 \\
21 \mathrm{R}-1,10-12 \\
24 \mathrm{R}-1,50-52 \\
28 \mathrm{R}-1,30-32 \\
29 \mathrm{R}-1,9-10 \\
30 \mathrm{R}-1,20-21 \\
34 \mathrm{R}-1,26-30 \\
37 \mathrm{R}-1,42-43\end{array}$ & $\begin{array}{l}676.69 \\
724.86 \\
773.80 \\
801.70 \\
839.20 \\
848.49 \\
859.10 \\
897.76 \\
926.92\end{array}$ & $\begin{array}{l}1 \\
1 \\
1 \\
1 \\
1 \\
1 \\
3 \\
1 \\
1 \\
1\end{array}$ & IIC & $\begin{array}{l}100 \\
100 \\
100 \\
100 \\
100 \\
100 \\
100 \\
100 \\
100\end{array}$ & & & \\
\hline $\begin{array}{l}139-858 \mathrm{~A}- \\
1 \mathrm{H}-1,39-41 \\
3 \mathrm{H}-6,37-41 \\
4 \mathrm{H}-6,23-27 \\
4 \mathrm{H}-6,23-27 \\
7 \mathrm{H}-2,40-43 \\
7 \mathrm{H}-2,40-43 \\
11 \mathrm{X}-\mathrm{CC}, 22-25 \\
11 \mathrm{X}-\mathrm{CC}, 22-25 \\
14 \mathrm{X}-1,6-8\end{array}$ & $\begin{array}{r}0.39 \\
19.54 \\
29.13 \\
29.13 \\
51.80 \\
51.80 \\
73.69 \\
73.69 \\
101.06\end{array}$ & $\begin{array}{l}3 \\
1 \\
1\end{array}$ & IIA & $\begin{array}{l}40 \\
21 \\
61 \\
70 \\
60 \\
63 \\
62 \\
66 \\
77\end{array}$ & $\begin{array}{l}28 \\
17 \\
39 \\
30 \\
40 \\
37 \\
38 \\
34 \\
23\end{array}$ & $\begin{array}{l}32 \\
62\end{array}$ & \\
\hline
\end{tabular}

a Ba-silicate or amphibole. Extensive epidotization and quartzification was observed in samples from Cores 139-857D-29R through -37R (848 to $926 \mathrm{mbsf}$ ). Large skeletal(?) crystals of sulfide also occur in this interval. Radiated crystals of epidote were observed in a fine-grained clayey interbed ( $897.8 \mathrm{mbsf})$ that has a high chlorite content, based on XRD.

Sulfide minerals, including pyrite and possibly others, occur also in veinlets along with anhydrite, quartz, and epidote. The veinlets in some cases cut parallel laminations of the turbidite.

\begin{tabular}{|c|c|c|c|c|c|c|c|}
\hline $\begin{array}{l}\text { Core, section, } \\
\text { interval }(\mathrm{cm})\end{array}$ & $\begin{array}{l}\text { Depth } \\
\text { (mbsf) }\end{array}$ & Fraction & Unit & $\begin{array}{l}\text { Chlorite } \\
\text { (\%) }\end{array}$ & $\begin{array}{c}\text { Hydromica } \\
(\%)\end{array}$ & $\begin{array}{l}\text { Smectite } \\
(\%)\end{array}$ & $\begin{array}{c}\text { Corrensite } \\
(\%)\end{array}$ \\
\hline $\begin{array}{l}14 X-1, ; 6-8 \\
14 X-C C, 18-20 \\
16 X-1,7-9 \\
17 X-1,32-34 \\
17 X-1, * 32-34 \\
18 X-2,71-73 \\
18 X-2, " 71-73 \\
20 X-1,96-98 \\
20 X-3,88-91 \\
21 X-2,60-62 \\
21 X-2,60-62 \\
21 X-3,19-21 \\
21 X-3, " 19-21 \\
24 X-1,40-42 \\
31 X-1,30-32 \\
31 X-1, " 30-32\end{array}$ & $\begin{array}{l}101.06 \\
101.18 \\
120.37 \\
130.22 \\
130.22 \\
141.81 \\
141.81 \\
159.96 \\
162.81 \\
170.70 \\
170.70 \\
171.60 \\
171.60 \\
198.00 \\
265.60 \\
265.60\end{array}$ & $\begin{array}{l}2 \\
1 \\
3 \\
1 \\
3 \\
1 \\
2 \\
3 \\
3 \\
1 \\
2 \\
1 \\
2 \\
1 \\
1 \\
2\end{array}$ & & $\begin{array}{l}79 \\
65 \\
80 \\
95 \\
84 \\
83 \\
84 \\
75 \\
85 \\
88 \\
83 \\
89 \\
89 \\
89 \\
88 \\
85\end{array}$ & $\begin{array}{r}21 \\
35 \\
20 \\
5 \\
16 \\
17 \\
16 \\
25 \\
15 \\
12 \\
17 \\
11 \\
11 \\
11 \\
12 \\
15\end{array}$ & & \\
\hline $\begin{array}{l}139-858 \mathrm{~B}- \\
1 \mathrm{H}-1,57-61 \\
1 \mathrm{H}-4,33-37 \\
1 \mathrm{H}-4,33-37 \\
2 \mathrm{H}-1,33-35 \\
2 \mathrm{H}-1,33-35 \\
2 \mathrm{H}-2,75-77 \\
2 \mathrm{H}-4,68-70 \\
2 \mathrm{H}-4, \cdot 96-98 \\
2 \mathrm{H}-4,96-98 \\
2 \mathrm{H}-6,46-48 \\
2 \mathrm{H}-6,46-48 \\
3 \mathrm{H}-1,126-128 \\
5 \mathrm{H}-2,17-19 \\
5 \mathrm{H}-4,14-18 \\
5 \mathrm{H}-4,14-18 \\
8 \mathrm{X}-1,13-15\end{array}$ & $\begin{array}{r}0.57 \\
4.83 \\
4.83 \\
7.53 \\
7.53 \\
9.45 \\
12.38 \\
12.66 \\
12.66 \\
15.16 \\
15.16 \\
17.96 \\
25.57 \\
28.54 \\
28.54 \\
32.83\end{array}$ & $\begin{array}{l}1 \\
1 \\
3 \\
1 \\
2 \\
1 \\
1 \\
1 \\
2 \\
1 \\
2 \\
3 \\
3 \\
1 \\
2 \\
1\end{array}$ & III & $\begin{array}{c}14 \\
12 \\
21 \\
10 \\
15 \\
26 \\
10 \\
25 \\
25 \\
83 \\
83 \\
25 \\
5 \\
\text { tr }\end{array}$ & $\begin{array}{r}9 \\
13 \\
33 \\
2 \\
6 \\
9\end{array}$ & $\begin{array}{l}77 \\
75 \\
46 \\
88 \\
79 \\
65\end{array}$ & $\begin{array}{r}75 \\
95 \\
100 \\
100\end{array}$ \\
\hline $\begin{array}{l}139-858 \mathrm{C}- \\
1 \mathrm{H}-1,112-116 \\
2 \mathrm{H}-2,26-28 \\
3 \mathrm{H}-1,100-102 \\
3 \mathrm{H}-6,48-50 \\
5 \mathrm{H}-3,46-48 \\
6 \mathrm{H}-1,114-116 \\
6 \mathrm{H}-1,114-116 \\
6 \mathrm{H}-2,46-48 \\
7 \mathrm{H}-1,16-19 \\
7 \mathrm{H}-1,16-19 \\
7 \mathrm{H}-1,115-118 \\
7 \mathrm{H}-1,115-118 \\
7 \mathrm{H}-2,124-127 \\
7 \mathrm{H}-3,123-127 \\
7 \mathrm{H}-3,123-127 \\
7 \mathrm{H}-4,10-14 \\
8 \mathrm{H}-1,12-14 \\
8 \mathrm{H}-1,28-30 \\
11 \mathrm{X}-1,71-73 \\
14 \mathrm{X}-\mathrm{CC}, 4-6\end{array}$ & $\begin{array}{r}1.12 \\
5.26 \\
14.00 \\
20.98 \\
26.96 \\
34.14 \\
34.14 \\
34.96 \\
41.66 \\
41.66 \\
42.65 \\
42.65 \\
44.24 \\
45.73 \\
45.73 \\
46.10 \\
46.62 \\
46.78 \\
55.21 \\
84.22\end{array}$ & $\begin{array}{l}1 \\
1 \\
2 \\
3 \\
1 \\
1 \\
2 \\
1 \\
1 \\
3 \\
1 \\
1 \\
3 \\
1 \\
1 \\
3 \\
1 \\
3 \\
1 \\
3 \\
1 \\
1\end{array}$ & IIIB & $\begin{array}{r}28 \\
28 \\
38 \\
38 \\
68 \\
100 \\
100 \\
100 \\
100 \\
90 \\
95 \\
91 \\
100 \\
100 \\
92 \\
87 \\
55 \\
94 \\
95 \\
98\end{array}$ & $\begin{array}{r}40 \\
17 \\
42 \\
23 \\
32 \\
\text { tr } \\
\text { tr } \\
\\
\text { tr } \\
10 \\
5 \\
9 \\
\text { tr } \\
\text { tr } \\
8 \\
13 \\
45 \\
6 \\
5 \\
2\end{array}$ & $\begin{array}{l}32 \\
55 \\
20 \\
39\end{array}$ & \\
\hline $\begin{array}{l}139-858 \mathrm{D}- \\
2 \mathrm{H}-3,95-97 \\
3 \mathrm{P}-1,17-19 \\
3 \mathrm{P}-1,17-19 \\
3 \mathrm{P}-1,17-19 \\
4 \mathrm{H}-3,65-69 \\
4 \mathrm{H}-3,65-69 \\
4 \mathrm{H}-4,100-104 \\
4 \mathrm{H}-6,124-126 \\
4 \mathrm{H}-6,124-126 \\
5 \mathrm{H}-\mathrm{CC}, 36-38\end{array}$ & $\begin{array}{l}13.25 \\
18.97 \\
18.97 \\
18.97 \\
22.19 \\
22.12 \\
24.04 \\
27.28 \\
27.28 \\
28.66\end{array}$ & $\begin{array}{l}1 \\
1 \\
2 \\
3 \\
1 \\
2 \\
2 \\
1 \\
3 \\
3\end{array}$ & $\begin{array}{c}\text { I } \\
\text { IIA }\end{array}$ & $\begin{array}{l}28 \\
68 \\
65 \\
62 \\
55 \\
59 \\
54 \\
50 \\
55 \\
79\end{array}$ & $\begin{array}{l}10 \\
32 \\
35 \\
38 \\
45 \\
41 \\
46 \\
50 \\
45 \\
21\end{array}$ & 62 & \\
\hline
\end{tabular}

Notes: Clay minerals as $100 \% .1=$ fraction less than $0.001 \mathrm{~mm} ; 2=$ fraction $0.001-$ $0.01 \mathrm{~mm} ; 3=$ nonseparated clay fraction from bulk samples; $\mathrm{tr}=$ trace amounts; ${ }^{\circ}=$ not used in diagrams.

We recognized the following secondary alterations of the igneous rocks recovered in Holes 857C and 857D (Table 4). Pyroxene is partially uralitized, with formation of fine-grained aggregates of leucoxene and sphene. The primary plagioclase is partially albitized and replaced by chlorite, epidote, and zeolite. Olivine is replaced by chlorite and leucoxene. Groundmass glass is chloritized. Veinlet minerals include chlorite, leucoxene, chalcedony, sulfides, epidote, quartz, and albite. Chlorite, epidote, quartz, and sulfide minerals fill pores and form amygdules. 
Table 2. Results of bulk X-ray diffraction mineralogy of sediments, Leg 139.

\begin{tabular}{|c|c|c|c|c|c|c|c|c|c|c|c|c|}
\hline $\begin{array}{l}\text { Core, section, } \\
\text { interval }(\mathrm{cm})\end{array}$ & $\begin{array}{l}\text { Depth } \\
\text { (m) }\end{array}$ & Unit & Q & F & $\mathrm{Am}$ & Cor & Anh & Gyp & Pyr & Ep & Cal & W \\
\hline \multicolumn{13}{|l|}{$139-856 \mathrm{~A}-$} \\
\hline $2 \mathrm{H}-3,67-69$ & 6.37 & & h+t+ & t+t & + & & + & & & & & \\
\hline $5 \mathrm{H}-7,26-30$ & 39.34 & IIA & t+r+ & H+ & & & + & & & & & \\
\hline $7 \mathrm{H}-4,133-137$ & 56.03 & & t+t+ & t+m & & & + & & & & & \\
\hline $7 \mathrm{H}-4,133-137$ & 56.03 & & t+t+ & +t+ & & & & & & & & \\
\hline $11 X-1,77-79$ & 86.97 & & H++ & +t+ & & & & & & & & \\
\hline $13 \mathrm{X}-1,108-111$ & 106.38 & IIC & H++ & +H+ & & & & & & & & \\
\hline $13 \mathrm{X}-4,94-98$ & 110.74 & & +++ & +++ & & & & & + & & & \\
\hline \multicolumn{13}{|l|}{ 139-856B- } \\
\hline $3 \mathrm{H}-1,108-110$ & 12.38 & & H+t & t+m & + & & & & & & & \\
\hline $3 \mathrm{H}-2,58-62$ & 13.38 & & h+t+ & +H+ & + & & + & + & & & & \\
\hline $3 \mathrm{H}-3,92-96$ & 15.22 & & t+t+ & H+ & + & & & & & & & \\
\hline $3 \mathrm{H}-4,62-66$ & 16.42 & & t+++ & t++ & & & + & & & & & \\
\hline $6 \mathrm{H}-4,95-97$ & 45.25 & & t+t+ & + & & & + & & & & & \\
\hline $7 \mathrm{H}-4,46-49$ & 54.26 & IIC & t+++ & + & & & & & & & & \\
\hline $8 \mathrm{H}-2,74-76$ & 61.04 & & +H++ & & & & & & & & & \\
\hline $12 X-4,106-110$ & 87.16 & & t+t+ & + & & & & & & & & \\
\hline $13 \times-4,84-88$ & 96.44 & & t+t+ & + & & & & & & & & \\
\hline $14 X-2,97-100$ & 103.27 & IID & ++t+ & & & & & & & & & \\
\hline $15 X-4,73-75$ & 115.63 & & ++++ & & & & & & & & & \\
\hline $139-857 \mathrm{C}$ & & & & & & & & & & & & \\
\hline $3 R-2,74-76$ & 68.74 & IIA & t+t+ & +++ & + & & & & & & & \\
\hline $12 \mathrm{R}-2,47-50$ & 145.47 & & ++++ & +t+ & & & & & & & & \\
\hline $15 \mathrm{R}-3,74-76$ & 176.24 & & ++++ & +++ & & & & & & & & \\
\hline $17 \mathrm{R}-2,112-116$ & 194.52 & & ++++ & +++ & & & & & & & & \\
\hline & 203.48 & & ++++ & +++ & & & & & & & & \\
\hline $\begin{array}{l}24 \mathrm{R}-1,44-46 \\
27 \mathrm{R}-2,81-86\end{array}$ & $\begin{array}{l}259.64 \\
286.41\end{array}$ & IIB & $\begin{array}{l}++++ \\
+++t\end{array}$ & $\stackrel{++}{+++}$ & & & & & & & & \\
\hline $31 \mathrm{R}-2,7-9$ & $\begin{array}{l}280.41 \\
324.27\end{array}$ & & $\begin{array}{l}t+++ \\
+++t\end{array}$ & +++ & & & & & & & & \\
\hline $33 \mathrm{R}-2,4-6$ & 333.94 & & t+++ & +t+ & & & & & & & & \\
\hline $36 \mathrm{R}-2,139-143$ & 348.99 & & ++++ & t++ & & & & & & & & \\
\hline $41 \mathrm{R}-3,48-50$ & 378.38 & & ++++ & +++ & & & & & & & & \\
\hline $43 \mathrm{R}-2,20-23$ & 386.4 & & ++++ & +++ & & & & & & & & \\
\hline $\begin{array}{l}51 \mathrm{R}-2,75-78 \\
65 \mathrm{R}-1,12-14\end{array}$ & $\begin{array}{l}425.65 \\
529.22\end{array}$ & IIC & $\begin{array}{l}++++ \\
+++\end{array}$ & $\begin{array}{l}+++ \\
+++\end{array}$ & & & & & & + & & \\
\hline $67 R-1,28-30$ & 548.68 & & +++ & ++ & & & & & & + & & \\
\hline 139-857D- & & & & & & & & & & & & \\
\hline $1 \mathrm{R}-2,19-22$ & 583.19 & & t+++ & + & & & & & & + & & \\
\hline $8 \mathrm{R}-1,5-7$ & 647.35 & & ++++ & + & & & & & & + & & \\
\hline $11 \mathrm{R}-1,49-51$ & 676.69 & & t+++ & +++ & & & & & & + & & \\
\hline $14 \mathrm{R}-1,1-4^{*}$ & 705.11 & & ++++ & ++ & & & & & & + & & \\
\hline $16 \mathrm{R}-1,36-38$ & 724.86 & & ++++ & +++ & & & & & & + & & \\
\hline $17 \mathrm{R}-1,45-48$ & 734.35 & & ++++ & ++t & & & & & & + & & \\
\hline $21 \mathrm{R}-1,119-121$ & 773.39 & & t+t+ & +++ & & & & & & + & & \\
\hline $21 \mathrm{R}-2,10-12$ & 773.8 & & ++++ & ++ & & & & & & + & & \\
\hline $22 \mathrm{R}-\mathrm{I}, 27-29$ & 782.07 & & ++++ & +++ & & & & & & + & & \\
\hline $24 \mathrm{R}-1,50-52$ & 801.7 & & ++++ & +++ & & & & & & + & & \\
\hline $27 \mathrm{R}-1,13-15$ & 829.73 & & ++++ & +++ & & & & & & + & & \\
\hline $28 \mathrm{R}-1,30-32$ & 839.2 & & ++++ & t++ & + & & & & & & & \\
\hline $29 \mathrm{R}-1,9-10$ & 848.49 & & n+t+ & t+m & & & & & & + & & \\
\hline $3 \mathrm{OR}-1,20-21$ & 859.1 & & ++++ & +H+ & & & & & & + & & \\
\hline $31 \mathrm{R}-1,13-15$ & 868.63 & & t+r+ & +H & & & & & & + & & \\
\hline $34 \mathrm{R}-1,26-30$ & 897.76 & & +H++ & +++ & & & & & & + & & \\
\hline $37 \mathrm{R}-1,42-43$ & 926.92 & & +h+t & + & & & & & & + & & \\
\hline 139-858A- & & & & & & & & & & & & \\
\hline $1 \mathrm{H}-1,39-41$ & 0.39 & 1 & n+t+ & + & & & & & & & & \\
\hline $3 \mathrm{H}-6,37-41$ & 19.54 & & t+h+ & + & & & & & & & & \\
\hline $4 \mathrm{H}-6,23-27$ & 29.13 & IIA & t+t+ & + & & & & & & & & \\
\hline $5 \mathrm{H}-3,32-37$ & 34.22 & & t+r+ & + & & & + & & & & & \\
\hline $11 \mathrm{X}-\mathrm{CC}, 22-25$ & 73.69 & & +H++ & + & & & + & + & & & + & \\
\hline $14 X-1,6-8$ & 101.06 & & +r++ & + & & & & & & & & \\
\hline 14X-CC, $16-20$ & 101.18 & ПIC & H+H & + & & & & & & & & \\
\hline $16 \times-1,7-9$ & 120.37 & & H+H & + & & & + & & & & & \\
\hline $17 \mathrm{X}-1,32-34$ & 130.22 & & H+H & ++ & & & H+ & + & & & & \\
\hline $18 \mathrm{X}-2,71-73$ & 141.81 & & $\mathrm{H+H}$ & + & & & H++ & + & & & & \\
\hline $20 X-1,96-98$ & 159.96 & & ++++ & + & & & & & & & & \\
\hline $20 X-3,88-91$ & 162.81 & & t+t+ & +t+ & & & + & + & & & & \\
\hline $21 \times-2,60-62$ & 170.7 & & t+r+ & + & & & + & + & & & & \\
\hline $21 \times-3,19-23$ & 171.6 & & +H+ & + & & & + & + & & & & \\
\hline $23 \mathrm{X}-1,97-99$ & 188.87 & & t+++ & t++ & & & + & + & & & & \\
\hline $24 \mathrm{X}-1,40-42$ & 198 & & t+++ & +t+ & & & & + & & & & \\
\hline $31 \times-1,30-32$ & 265.6 & & $\mathrm{H+H}$ & +++ & & & + & + & & & & \\
\hline $39 \mathrm{R}-\mathrm{CC}, 15-17$ & 332.55 & & H+t+ & + & & & & & & & & \\
\hline 139-858B- & & & & & & & & & & & & \\
\hline $1 \mathrm{H}-1,57-61$ & 0.57 & III & n+++ & +++ & & & & & & & & \\
\hline $1 \mathrm{H}-4,33-37$ & 4.83 & & $\mathrm{H+H}$ & + & + & & & & + & & & \\
\hline $2 \mathrm{H}-2,75-77$ & 9.45 & & $\mathrm{H+H} 2$ & H+ & & & + & & + & & & \\
\hline $2 \mathrm{H}-4,68-70$ & 12.38 & I & & & & & t+++ & + & + & & & \\
\hline $2 \mathrm{H}-4,96-98$ & 12.66 & & H+t+ & + & & & t+t & + & + & & & \\
\hline $2 \mathrm{H}-6,46-48$ & 15.16 & & ++1+ & + & & & + & & & & & \\
\hline $5 \mathrm{H}-4,14-18$ & 28.54 & IIB & & & & +t+ & & & & & & \\
\hline $8 \mathrm{X}-1,13-15$ & 32.83 & IID & ++++ & & & & & & & & & \\
\hline
\end{tabular}


Table 2 (continued).

\begin{tabular}{|c|c|c|c|c|c|c|c|c|c|c|c|c|}
\hline $\begin{array}{l}\text { Core, section, } \\
\text { interval }(\mathrm{cm})\end{array}$ & $\begin{array}{l}\text { Depth } \\
\text { (m) }\end{array}$ & Unit & Q & $\mathrm{F}$ & Am & Cor & Anh & Gyp & Pyr & Ep & Cal & W \\
\hline \multicolumn{13}{|l|}{$139-858 \mathrm{C}$} \\
\hline IH-1, 112-116 & 1.12 & I & +++ & + & & & & & & & + & \\
\hline $3 \mathrm{H}-6,48-50$ & 20.98 & & +++ & + & & & + & + & + & & & \\
\hline $5 \mathrm{H}-3,46-48$ & 26.96 & & ++ & + & & & + & + & + & & & \\
\hline $6 \mathrm{H}-3,34-36$ & 36.34 & & ++ & + & & & +++ & + & + & & & \\
\hline $7 \mathrm{H}-1,16-19$ & 41.66 & & +++ & + & & & & & + & & & \\
\hline $7 \mathrm{H}-1,115-118$ & 42.65 & IIIB & +++ & + & & & & & & & & \\
\hline $7 \mathrm{H}-2,124-127$ & 44.24 & & +++ & + & + & & & & + & & & \\
\hline $7 \mathrm{H}-3,123-127$ & 45.73 & & +++ & + & & & & + & + & & & \\
\hline $8 \mathrm{H}-1,12-14$ & 46.62 & & +++ & + & & & + & + & + & & + & \\
\hline $8 \mathrm{H}-1,28-30$ & 46.78 & & +++ & + & & & & & + & & & \\
\hline $11 X-1,71-73$ & 55.21 & IIC & +++ & + & & & & & & & & \\
\hline $14 \mathrm{X}-\mathrm{CC}, 4-6$ & 84.22 & & +++ & + & & & & & + & & & \\
\hline \multicolumn{13}{|l|}{ 139-858D- } \\
\hline $2 \mathrm{H}-3,95-98$ & 13.25 & I & $t++$ & + & & & & & & & & \\
\hline $3 P-1,17-19$ & 18.97 & & h+t+ & + & & & & + & & & & \\
\hline $4 \mathrm{H}-3,65-69$ & 22.19 & & +++ & + & & & & + & & & & \\
\hline $4 \mathrm{H}-4,100-104$ & 24.04 & IIA & +++ & + & & & + & + & & & & \\
\hline $4 \mathrm{H}-6,124-126$ & 27.28 & & +++ & & & & & & & & & \\
\hline 5H-CC, 36-38 & 28.66 & & 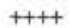 & & & & & & + & & & + \\
\hline $6 \mathrm{X}-1,25-27$ & 29.05 & IID & $t+t+$ & & & & & & + & & & + \\
\hline
\end{tabular}

Note: $++++=$ dominant $+++=$ major, $++=$ minor,$+=$ trace; $*=$ talc $($ trace $) ; \mathrm{Q}=$ quartz, $\mathrm{F}=$ feldspars, Am = amphibole, $\mathrm{Cor}=$ corrensite-like mineral, $\mathrm{Anh}=$ anhydrite, $\mathrm{Gyp}=$ gypsum, $\mathrm{Pyr}=$ pyrite, $\mathrm{Ep}=$ epidote, $\mathrm{Cal}=$ calcite, $\mathrm{W}=$ wairacite .

Chlorite with $\mathrm{Fe}$ predominating over $\mathrm{Mg}$ is the most common alteration product. Sometimes, especially in the lower part of the igneous complex, corrensite-like minerals occur together with chlorite.

On the whole Zone III sediments and the igneous rocks interbedded with them have undergone alteration in the greenschist facies of metamorphism. The sediments are chloritized (Fe chlorite), epidotized, and quartzified. Presumably there are newly-formed feldspars in these sediments. The igneous rocks are extensively chloritized, and epidote, quartz, albite, and uralite also occur.

\section{Site 858}

Lithologic Unit I (Davis, Mottl, Fisher, et al., 1992) is composed of silty clay of Holocene to late Pleistocene age. Lithologic Unit II is characterized by alternating hemipelagic and turbiditic sediments and is generally correlative to lithologic Unit II at the previous Leg 139 sites. At Site 858, lithologic Unit II is divided into four subunits. Subunit IIA is nonaltered to weakly altered, Subunit IIC is moderately to well indurated and altered, and Subunit IID is completely silicified, commonly fractured, and contains abundant authigenic silica and sulfide minerals.

These three subunits are lithologically similar to Subunits IIA, IIC, and IID at Site 856. Subunit IIB is a brecciated, anhydrite, and carbonate-rich sequence that contains semimassive sulfide. Subunit IIB is in a stratigraphic position similar to that of the slumped (?) Subunit IIB at Site 856. Lithologic Unit III consists of metalliferous, oxidized surficial sediments. Lithologic Unit IV consists of stratabound semimassive sulfide. Lithologic Unit V consists of mafic extrusive rock recovered at the base of Holes $858 \mathrm{~F}$ and $858 \mathrm{G}$.

\section{Hole 858 A}

Mineralogical data distinguishes three zones of hydrothermal alteration in sediments from Hole 858A.

Zone I (0-21.40 mbsf) coincides with lithologic Unit I and contains abundant authigenic smectite. The zone is a smectite-bearing (Fig. 8), or smectite-dominated (19.5 mbsf) hemipelagic silty clay.

Zone II ( 21.40 to $315 \mathrm{mbsf}$ ) coincides with lithologic Subunits IIA and IIC and contains among authigenic minerals mainly pyrite, chlorite, and anhydrite, as well as calcite in the upper part of the zone, and epidote and quartz in the lower part of the zone. Mineralogical data show that Zone II is divided into Subzones IIA (21.40 to $120 \mathrm{mbsf}$ ) and IIB (120 to $315 \mathrm{mbsf}$ ).
Subzone IIA is characterized by detrital quartz predominating over feldspars, based on bulk XRD (Table 2 ) and by approximately uniform ratios of mica (40\%) to Fe chlorite $(60 \%)$ in the clay fraction (Fig. 8). Trace amounts of anhydrite occur throughout and gypsum also was found (Table 2).

This subzone is characterized in shipboard descriptions as an interval with carbonate concretions. These occur together with anhydrite and pyrite concretions. In thin sections we observed recrystallized foraminifers covered by rusty Fe-oxide films, some of which look like micronodules in the coarse fraction. Calcite micronodules occur as detrital grains and as ellipsoidal nodule-like segregations, the latter being possibly of authigenic (hydrothermal) origin. Zoisite or clinozoisite replaces sodium plagioclase grains.

The coarse heavy fraction (Table 3 ) contains high percentages of pyrite and $\mathrm{Fe}$-oxides; epidote, barite, and garnet are common transparent minerals. In the light fraction downhole trends of increasing quartz and decreasing feldspar were observed. Both muscovite and biotite decrease downward.

In thin sections, sandy layers are seen to contain amphibole, quartz, and feldspar. The sediment is partially cemented by carbonate. Carbonate also occurs as recrystallized foraminiferal tests. Epidoteclinozoisite occurs commonly in the cement. Leucoxene is present.

Subzone IIB as a whole is characterized by a high quartz content that increases downward, by variable feldspar content, and by chlorite apparently prevailing over mica. Most significant, however, is the high and variable anhydrite and gypsum content (Table 2). Fe chlorite prevails strongly over hydromica in the clay fraction, with some oscillations in their relative amounts (Table 1, Fig. 8).

The interval below 175 mbsf contains low concentrations of sulfates. Quartzification (quartz cementation) is more extensive. Carbonate is absent. Chloritization of mica is accompanied by pyritization, leading to formation of chlorite aggregates with abundant disseminated finely crystalline pyrite.

Zone III ( 315 to $332.6 \mathrm{mbsf}$ ) coincides with lithologic Subunit IID and contains among authigenic minerals sulfide, chlorite, quartz, and wairakite-analcime veinlets.

\section{Hole $858 B$}

Three zones of hydrothermal alteration were distinguished in Hole $858 \mathrm{~B}$. The semimassive sulfides are not included in our study.

Zone I ( 0 to about $10 \mathrm{mbsf}$ ) coincides with lithologic Units I and III, characterized by the presence or even domination of smectites in 
Table 3. Coarse fraction $(0.1-0.05 \mathrm{~mm})$ mineralogy.

\begin{tabular}{|c|c|c|c|c|c|c|c|c|c|c|c|c|}
\hline $\begin{array}{l}\text { Core, section } \\
\text { interval }(\mathrm{cm})\end{array}$ & Py & Iox & Ep & $\mathrm{Gr}$ & Zrk & Sph & Ap & $\mathrm{Px}$ & Am & Bar & Chl & Anh \\
\hline $139-856 \mathrm{~A}-$ & & & & & & & & & & & & \\
\hline $1 \mathrm{H}-1,30-33^{\circ}$ & 1.4 & 3.1 & - & - & 0.2 & - & - & - & - & - & - & - \\
\hline $2 \mathrm{H}-3,67-69^{\circ}$ & 5.4 & 3.4 & . & - & - & - & - & - & - & - & - & - \\
\hline $2 \mathrm{H}-2,45-49$ & 22.2 & 5.9 & - & 0.3 & $=$ & - & - & - & 1.0 & - & - & - \\
\hline $4 \mathrm{H}-6,6-10^{\circ}$ & - & 6.3 & - & - & - & - & - & - & - & - & - & - \\
\hline $5 \mathrm{H}-7,26-30$ & 76.4 & 8.7 & 13.2 & 0.2 & 0.2 & 0.2 & 0.5 & - & - & - & 0.2 & - \\
\hline $6 \mathrm{H}-5,84-88$ & 52.3 & 40.6 & 0.8 & 0.4 & 0.8 & 0.8 & 0.4 & 1.6 & 1.5 & - & - & - \\
\hline $8 \mathrm{H}-4,82-85^{\circ}$ & 1.2 & 1.2 & 51.4 & 0.3 & 0.3 & $\because$ & - & 0.3 & 0.3 & - & - & - \\
\hline $9 \mathrm{H}-3,56-59$ & 11.9 & 79.9 & 5.4 & 0.3 & - & - & 0.3 & 0.3 & 0.3 & 1.0 & 0.3 & - \\
\hline $10 X-1,55-57$ & 60.4 & 31.6 & 6.8 & - & - & - & 0.3 & - & - & - & - & 0.9 \\
\hline $13 \times-3,93-97$ & 72.4 & 9.8 & 8.7 & 0.5 & - & - & 0.3 & - & - & 0.5 & 0.3 & - \\
\hline \multicolumn{13}{|l|}{ 139-856B- } \\
\hline IH-1, 33-37 & 76.0 & 18.9 & 7.6 & - & - & - & - & - & - & 2.5 & - & - \\
\hline $2 \mathrm{H}-3,106-110$ & 92.3 & 2.2 & 1.5 & - & - & - & - & - & 3.7 & - & - & 0.4 \\
\hline $3 \mathrm{H}-1,108-112$ & 60.9 & 2.7 & - & - & - & - & - & - & - & - & - & - \\
\hline $3 \mathrm{H}-2,58-62$ & 12.6 & 2.8 & 0.2 & - & - & - & - & 0.2 & - & - & - & - \\
\hline $3 \mathrm{H}-3,92-96$ & 95.7 & 2.8 & 0.9 & . & - & - & - & 0.3 & . & - & - & - \\
\hline $3 \mathrm{H}-4,62-66$ & 98.5 & $\because$ & 0.6 & - & - & - & - & 0.6 & - & - & - & - \\
\hline $4 \mathrm{H}-4,67-68$ & 95.5 & 1.8 & 1.4 & - & 0.3 & - & - & - & - & - & - & 0.7 \\
\hline $6 \mathrm{H}-4,95-97$ & 83.4 & 14.6 & 1.4 & 0.7 & - & - & - & - & - & - & - & - \\
\hline $7 \mathrm{H}-4,46-49$ & 90.9 & 4.6 & . & 0.9 & 0.9 & - & - & 0.3 & 1.1 & 0.3 & 0.6 & - \\
\hline $9 X-1,55-59$ & 89.5 & 2.1 & 0.5 & 1.6 & 0.8 & - & 4.2 & - & 0.5 & - & 0.3 & 0.5 \\
\hline $\begin{array}{l}12 X-4.106- \\
110\end{array}$ & 80.1 & 14.3 & 0.8 & 1.6 & 0.8 & - & - & 0.8 & - & 0.8 & - & - \\
\hline $13 X-1,69-73$ & 90.1 & 6.3 & 1.7 & 0.6 & - & - & 0.3 & - & - & - & - & 0.6 \\
\hline \multicolumn{13}{|l|}{ 139-857A- } \\
\hline $2 \mathrm{H}-2,8-10$ & 88.0 & 4.2 & 1.2 & - & - & - & - & 0.4 & 2.5 & $\therefore$ & 1.2 & - \\
\hline $6 \mathrm{H}-3,40-44$ & 96.7 & 0.7 & - & 0.2 & - & - & - & 0.2 & 0.6 & 0.7 & - & - \\
\hline $7 \mathrm{H}-4,30-34$ & 54.6 & 28.4 & 1.5 & - & - & - & - & 1.0 & 3.0 & 4.5 & 3.0 & - \\
\hline \multicolumn{13}{|l|}{$139-857 \mathrm{C}$ - } \\
\hline IR-1, 103-105 & 76.3 & 1.2 & 13.7 & 0.4 & 1.0 & - & 0.6 & - & 5.4 & 0.2 & 0.2 & - \\
\hline $2 \mathrm{R}-2,24-25$ & 74.0 & 13.6 & 0.9 & - & - & . & - & - & - & - & 5.8 & 1.9 \\
\hline $10 \mathrm{R}-1,72-74$ & 69.7 & 13.8 & 2.0 & 0.6 & - & - & 1.3 & - & 3.2 & - & - & - \\
\hline $15 \mathrm{R}-3,74-76$ & 13.3 & - & 2.0 & - & - & - & - & - & - & 3.4 & 1.5 & - \\
\hline $\begin{array}{l}\text { 17R-2, 112- } \\
116\end{array}$ & 23.9 & 1.3 & 49.8 & 0.6 & 0.6 & 3.8 & 3.0 & - & 0.8 & 2.5 & 0.4 & - \\
\hline \multicolumn{13}{|l|}{$139-858 \mathrm{~A}$ - } \\
\hline $3 \mathrm{H}-6,37-41$ & 91.6 & 2.3 & - & - & - & - & - & 0.6 & 1.7 & 0.8 & - & - \\
\hline $4 \mathrm{H}-6,23-27$ & 81.8 & 3.4 & 0.5 & - & - & - & - & - & 1.1 & - & - & 0.3 \\
\hline $5 \mathrm{H}-3^{\circ}, 32-37$ & - & - & 0.5 & - & - & - & - & - & - & 2.1 & - & - \\
\hline $7 \mathrm{H}-2^{*}, 40-43$ & 4.0 & - & - & - & - & - & - & - & - & - & - & - \\
\hline $11 \mathrm{H}-\mathrm{CC}, 22-25$ & 89.3 & 7.8 & 5.6 & 0.7 & . & - & - & - & - & 0.2 & 0.2 & - \\
\hline $\begin{array}{l}14 X-1 C C, 18- \\
20\end{array}$ & 89.6 & 11.9 & 3.1 & - & - & 1.0 & - & - & - & 1.7 & - & - \\
\hline $17 X-1,32-34$ & 7.8 & 5.4 & 36.2 & 15.4 & 3.2 & 0.2 & 8.5 & 0.5 & 0.7 & 1.0 & 1.0 & 7.5 \\
\hline \multicolumn{13}{|l|}{$139-858 \mathrm{~B}-$} \\
\hline $1 \mathrm{H}-4^{*}, 33-37$ & 5.5 & - & - & - & - & - & - & - & - & 3.3 & - & - \\
\hline $2 \mathrm{H}-6,46-48$ & 86.9 & 9.4 & 0.6 & - & . & - & - & . & - & 0.3 & - & - \\
\hline $5 \mathrm{H}-4,14-18$ & 98.2 & - & - & - & - & 1.6 & - & - & - & 0.2 & - & - \\
\hline \multicolumn{13}{|l|}{$139-858 \mathrm{C}$} \\
\hline $1 \mathrm{H}-1,112-116$ & 92.9 & 2.7 & 1.5 & . & - & - & - & 0.3 & 0.9 & 0.6 & - & - \\
\hline $2 \mathrm{H}-2,26-28$ & 89.5 & 4.6 & 1.5 & - & - & - & 0.5 & 0.7 & 1.0 & 1.0 & - & - \\
\hline $5 \mathrm{H}-3,46-48$ & 96.1 & 1.4 & 1.7 & - & - & - & - & - & . & - & - & - \\
\hline $6 \mathrm{H}-2,46-48$ & 97.5 & - & 0.2 & - & . & - & 0.7 & - & - & 0.2 & - & - \\
\hline $7 \mathrm{H}-1,16-19$ & 92.2 & 5.1 & - & - & - & - & - & . & - & 0.7 & - & - \\
\hline $7 \mathrm{H}-1,115-118$ & 86.8 & 10.6 & 2.2 & - & - & - & - & - & - & 0.4 & - & - \\
\hline $7 \mathrm{H}-2,121-127$ & 95.9 & - & - & . & - & - & - & - & - & 3.1 & - & - \\
\hline $7 \mathrm{H}-4,10-14$ & 90.1 & - & - & - & - & - & 0.6 & - & - & 2.0 & - & - \\
\hline $14 X-2,4-6$ & 96.3 & - & 2.5 & 0.3 & - & - & - & - & - & - & - & - \\
\hline \multicolumn{13}{|l|}{$139-858 \mathrm{D}-$} \\
\hline $4 \mathrm{H}-3,65-69$ & 94.7 & 1.8 & 0.2 & - & - & - & - & - & - & 3.1 & - & - \\
\hline $4 \mathrm{H}-6,124-126$ & 91.4 & 1.8 & - & 0.4 & - & - & - & 0.9 & - & 0.2 & - & - \\
\hline
\end{tabular}

the clay fraction (Table 1, Fig. 9). The upper $2 \mathrm{~m}$ is "metalliferous" clay (green, greenish gray, greenish black to dark bluish green, with brownish yellow oxidized surface layer). We observed nontronite aggregates in a smear-slide; smectite dominates in the clay fraction (Fig. 9). In smear slides the smectitic clay contains detrital quartz, feldspar, and minor mica, with rare epidote-clinozoisite and leucoxene, the latter in small aggregates with rutile. Finely crystalline disseminated anhydrite and pyrite are present in minor amounts.

Zone II (10 to about $30 \mathrm{mbsf}$ ) coincides with lithologic Units I (lower part, below Unit IV), IV, and Subunit IIB and contains among authigenic minerals mainly pyrite, corrensite-like mineral, chlorite, and anhydrite. Bulk XRD data shows high quartz content and trace amounts of feldspar (Table 2). The major change in clay mineralogy is the disappearance of mica and the appearance of $\mathrm{Mg}$-rich mixedlayer smectite/chlorite (corrensite-like mineral) (Table 1, Fig. 9). In thin section we observed authigenic well-crystallized chlorite (or corrensite) clay with abundant pyrite, both disseminated finely crystalline and as aggregates of large crystals (pseudomorphs after anhydrite?). A radiated aggregate of elongated anhydrite crystals includes euhedral cubic pyrite and is partially replaced by gypsum.

Zone III ( 30 to $38.6 \mathrm{mbsf}$ ) coincides with lithologic Subunit IID. A single Sample 139-858B-8X-1, 46-48 cm (32.83 mbsf) represents 
Table 3 (continued).

B. Light minerals.

\begin{tabular}{|c|c|c|c|c|c|c|c|c|c|c|}
\hline $\begin{array}{l}\text { Core, section, } \\
\text { interval }(\mathrm{cm})\end{array}$ & $\mathrm{Qz}$ & Fs & $\mathrm{Bi}$ & $\mathrm{Mu}$ & Chl & Anh & Gyp & $\mathrm{Ca}$ & C.A. & $\mathrm{Ze}$ \\
\hline \multicolumn{11}{|l|}{$139-856 \mathrm{~A}-$} \\
\hline $1 \mathrm{H}-1,30-33$ & 39.1 & 11.2 & 2.1 & 2.8 & 0.7 & - & - & 1.4 & 37.0 & 1.0 \\
\hline $2 \mathrm{H}-3,67-69$ & 7.4 & 4.3 & 3.4 & 3.4 & 2.3 & - & - & 46.0 & 21.2 & 1.1 \\
\hline $2 \mathrm{H}-2,45-49$ & 9.2 & 1.8 & - & - & $\because$ & - & - & 57.3 & 2.0 & 0.3 \\
\hline $4 \mathrm{H}-6,6-10$ & 5.4 & 2.7 & - & - & - & - & - & 83.0 & 2.6 & - \\
\hline $5 \mathrm{H}-7,26-30$ & 9.1 & 2.6 & 1.6 & 0.5 & - & - & - & 42.1 & 44.2 & - \\
\hline $6 \mathrm{H}-5,84-88$ & 1.1 & 0.8 & $\therefore$ & 0.4 & - & - & - & 97.3 & 0.4 & - \\
\hline $8 \mathrm{H}-4,82-85$ & 36.2 & 17.6 & 0.9 & 0.9 & 0.3 & - & - & 4.9 & 34.1 & - \\
\hline $9 \mathrm{H}-3,56-59$ & 43.2 & 11.5 & 0.4 & $\because$ & 0.4 & - & - & 4.2 & 39.1 & 0.8 \\
\hline $10 \times-1,55-57$ & 45.7 & 21.0 & 0.7 & 1.4 & 1.4 & - & 0.2 & 15.7 & 19.4 & 0.5 \\
\hline $13 X-3,93-97$ & 35.5 & 19.0 & 6.1 & 5.1 & 0.6 & - & - & 0.6 & 32.1 & 0.6 \\
\hline \multicolumn{11}{|l|}{ 139-856B- } \\
\hline $1 \mathrm{H}-1,33-37$ & 7.1 & 3.4 & - & - & 0.4 & - & - & 81.1 & 4.2 & - \\
\hline $2 \mathrm{H}-3,106-110$ & 17.3 & 7.6 & 1.1 & 1.1 & - & - & - & 3.6 & 67.5 & 1.8 \\
\hline $3 \mathrm{H}-1,108-112$ & 4.7 & 1.5 & 0.4 & 0.4 & - & - & - & 17.2 & 11.8 & 0.4 \\
\hline $3 \mathrm{H}-2,58-62$ & 5.1 & 6.0 & 1.6 & 1.4 & 0.2 & - & - & 6.2 & 53.7 & - \\
\hline $3 \mathrm{H}-3,92-96$ & 5.7 & 3.1 & 1.4 & 2.0 & 1.0 & - & - & 4.7 & 80.4 & 1.7 \\
\hline $3 \mathrm{H}-4,62-66$ & 40.8 & 10.2 & 2.6 & 1.6 & 0.4 & - & - & 4.0 & 38.9 & 1.2 \\
\hline $4 \mathrm{H}-4,67-68$ & 13.1 & 2.6 & 1.0 & 0.5 & - & - & - & 12.6 & 69.7 & - \\
\hline $6 \mathrm{H}-4,95-97$ & 10.3 & 4.4 & 1.5 & 5.2 & 0.7 & - & 0.4 & 1.5 & 74.5 & 1.5 \\
\hline $7 \mathrm{H}-4,46-49$ & 42.3 & 16.0 & 1.5 & 1.2 & - & - & - & 1.2 & 37.2 & $\therefore$ \\
\hline $9 X-1,55-59$ & 41.3 & 13.2 & 3.7 & 1.7 & 1.4 & - & 0.3 & 2.0 & 35.5 & 0.9 \\
\hline $\begin{array}{l}12 X-4,106- \\
110\end{array}$ & 12.6 & 3.1 & 2.3 & 0.4 & $\because$ & - & - & 2.7 & 80.5 & $\because$ \\
\hline $13 X-1,69-73$ & 27.7 & 11.2 & 0.9 & 0.6 & 0.3 & - & - & 1.8 & 57.5 & - \\
\hline \multicolumn{11}{|l|}{ 139-857A- } \\
\hline $2 \mathrm{H}-, 8-10$ & 8.2 & 7.9 & 2.2 & 1.0 & 1.0 & - & - & 63.4 & 13.0 & 1.0 \\
\hline $6 \mathrm{H}-3,40-44$ & 16.3 & 7.7 & 15.8 & 7.0 & - & - & - & 5.5 & 17.8 & - \\
\hline $7 \mathrm{H}-4,30-34$ & 18.6 & 9.3 & 3.8 & 1.6 & - & 1.1 & 1.6 & 46.6 & 15.8 & 0.5 \\
\hline \multicolumn{11}{|l|}{ 139-857C- } \\
\hline 1R-1, 103-105 & 18.2 & 19.8 & 4.3 & 1.0 & 1.3 & - & - & 2.3 & 17.3 & - \\
\hline $2 \mathrm{R}-2,24-25$ & 16.0 & 10.6 & 1.5 & 0.3 & - & 1.5 & - & 39.2 & 27.9 & 1.5 \\
\hline $10 \mathrm{R}-1,72-74$ & 22.2 & 11.8 & 2.6 & 1.5 & 0.7 & - & - & 26.5 & 28.3 & - \\
\hline $15 R-3,74-76$ & 26.1 & 8.4 & - & - & - & - & - & 10.3 & 22.7 & - \\
\hline $\begin{array}{l}\text { 17R-2, } 112- \\
116\end{array}$ & 44.0 & 23.2 & 2.4 & 3.1 & - & - & - & 2.0 & 22.2 & - \\
\hline \multicolumn{11}{|l|}{$139-858 \mathrm{~A}-$} \\
\hline $3 \mathrm{H}-6,37-41$ & 18.0 & 10.8 & 3.4 & 0.9 & - & - & - & 43.6 & 18.1 & - \\
\hline $4 \mathrm{H}-6,23-27$ & 14.2 & 8.1 & 0.4 & 1.1 & - & 1.1 & - & 51.5 & 14.2 & - \\
\hline $5 \mathrm{H}-3,32-37$ & 11.2 & 4.4 & 1.0 & 1.4 & - & - & - & 58.5 & 23.5 & - \\
\hline $7 \mathrm{H}-2,40-43$ & 7.3 & 2.7 & - & $\because$ & - & - & - & 70.6 & 10.8 & - \\
\hline $11 \mathrm{H}-\mathrm{CC}, 22-25$ & 38.6 & 18.3 & 1.4 & - & - & - & . & 3.2 & 16.4 & 0.5 \\
\hline $\begin{array}{l}14 \mathrm{X}-1 \mathrm{ICC}, 18- \\
20\end{array}$ & 12.8 & 7.2 & $\cdot$ & 2.2 & - & - & - & 2.5 & 47.2 & - \\
\hline $17 \mathrm{X}-1,32-34$ & 31.7 & 16.1 & - & - & 1.5 & 8.0 & - & 0.3 & 40.3 & - \\
\hline \multicolumn{11}{|l|}{ 139-858B- } \\
\hline $1 \mathrm{H}-4,33-37$ & 16.7 & 6.4 & 1.0 & 3.3 & - & 0.5 & 0.2 & 4.3 & 54.8 & 1.3 \\
\hline $2 \mathrm{H}-6,46-48$ & 62.3 & 15.8 & 1.9 & - & 0.3 & - & - & 2.2 & 15.0 & - \\
\hline $5 \mathrm{H}-4,14-18$ & 4.2 & 1.2 & 0.4 & 0.8 & - & - & - & 2.3 & 89.2 & $=$ \\
\hline \multicolumn{11}{|l|}{$139-858 \mathrm{C}$ - } \\
\hline $1 \mathrm{H}-1,112-116$ & 5.6 & 4.0 & 1.2 & 0.9 & - & - & - & 85.2 & - & 0.6 \\
\hline $2 \mathrm{H}-2,26-28$ & 6.5 & 1.5 & 0.7 & - & - & - & - & 91.3 & - & - \\
\hline $5 \mathrm{H}-3,46-48$ & 58.5 & 15.3 & - & - & . & . & - & 1.3 & 14.1 & - \\
\hline $6 \mathrm{H}-2,46-48$ & 28.8 & 6.9 & 0.6 & 2.2 & 0.4 & - & - & 0.9 & 50.6 & 0.4 \\
\hline $7 \mathrm{H}-1,16-19$ & 17.5 & 9.9 & 2.4 & 3.3 & - & - & - & - & 66.3 & - \\
\hline $7 \mathrm{H}-1,115-118$ & 5.9 & 4.4 & 1.7 & 1.7 & - & - & . & 4.5 & 73.8 & - \\
\hline $7 \mathrm{H}-2,121-127$ & - & - & 0.9 & 1.5 & - & - & - & 3.1 & 94.5 & - \\
\hline $7 \mathrm{H}-4,10-14$ & 12.3 & 4.1 & 0.7 & 1.3 & - & 0.2 & . & 0.2 & 76.1 & - \\
\hline $14 X-2,4-6$ & 66.0 & 14.7 & 0.9 & 2.4 & - & - & - & 1.6 & 14.4 & - \\
\hline
\end{tabular}

Notes: ${ }^{*}=$ minerals counted without separation into heavy and light fractions. $\mathrm{Py}=$ pyrite, Iox $=$ iron oxides, $\mathrm{Ep}=$ epidote, $\mathrm{Gr}=$ gamet, $\mathrm{Zrk}=$ zircon, $\mathrm{Sph}=$ sphene, $\mathrm{Ap}=$ apatite, $\mathrm{Px}=$ pyroxenes, $\mathrm{Am}=$ amphibole, $\mathrm{Bar}=$ barite, $\mathrm{Chl}=$ chlorite, $\mathrm{Anh}=$ anhydrite, $\mathrm{Qz}=$ quartz, $\mathrm{Fs}=$ feldspars, $\mathrm{B}$ = biotite, $\mathrm{Mu}=$ muscovite, $\mathrm{Chl}=$ chlorite, $\mathrm{Anh}=$ anhydrite, $\mathrm{Gyp}=$ gypsum, $\mathrm{Ca}=$ calcite (mainly biogenic), but with minor sparry calcite, C.A. = unidentified clay aggregates, $\mathrm{Ze}=$ zeolites. Other rare minerals not included in the table are (heavy fraction): tremolite and/or actinolite, black opaques, olivine, rutile, leucoxene, carbonate(?); (light fraction): chalcedony, opal A (siliceous microfossils), volcanic glass, talc(?). - = mineral not found.

the zone and is a chlorite-quartz metamorphic rock according to bulk XRD (Tables 1 and 2) and thin section description. This is consistent with the shipboard XRD data, which show abundant quartz and rather high chlorite without other minerals. The clay fraction in our sample is composed of pure Fe chlorite (Table 1, Fig. 9). In thin section the rock is composed of elongated quartz crystals with interstitial chlorite.

\section{Hole $858 \mathrm{C}$}

Three zones of hydrothermal alteration can be distinguished based on different authigenic (hydrothermal) mineral associations.

Zone I ( 0 to about $20 \mathrm{mbsf}$ ) coincides approximately with lithostratigraphic Unit I and is composed of hemipelagic silty clay with up 
Table 4. Brief petrographic description of analyzed igneous rocks and secondary minerals, Leg 139.

\begin{tabular}{lll}
\hline $\begin{array}{l}\text { Core, section } \\
\text { interval }(\mathrm{cm})\end{array}$ & $\begin{array}{l}\text { Depth } \\
(\mathrm{mbsf})\end{array}$ & Description \\
\hline
\end{tabular}

$139-856 \mathrm{~A}$

14X-1, 34-38 $\quad 114.84$ Pyroxene microdoleritic basalt. Phenocrysts: clinopyroxene (up to $1 \mathrm{~mm}$ ) sparse olivine, Groundmass: non-oriented lathes and microliths of plagioclase $(0.2-1 \mathrm{~mm})$ - labrador $\left(\mathrm{An}_{55}\right)$. Among microliths there are individuals up to andesine $\left(\mathrm{An}_{35}\right)$. Clinopyroxene in interstices. Opaques: magnetite, leucoxene, spinel, chalcopyrite.

Alteration is low. Olivine is replaced by iddingsite and talc. Plagioclase and clinopyroxene are partially replaced by prehnite and chlorite. Zeolite is possibly formed on the plagioclase.

139-857C-

$59 \mathrm{R}-1,20-22$

$59 \mathrm{R}-2,57-59$

$60 \mathrm{R}-1,130-132$

61R-2, 8-10

$62 \mathrm{R}-1,70-72$

$62 \mathrm{R}-2,40-42$

$63 \mathrm{R}-1,50-52$

64R-1, 18-20

$68 \mathrm{R}-2,69-71$

139-857D-

$3 R-2,86-88$

4R-2, 53-56

$8 \mathrm{R}-1,5-7$

12R-1, 106-108

$2 \mathrm{R}-2,17-19$

17R-1, 45-48

17R-3. 72-74
471.30 Plagioclase-pyroxene dolerite, fine-grained with ophytic texture Alteration is strong (up to $50 \%$ of rock is altered). Pyroxene and glass of groundmass are chloritized. Veinlets: chalcedony in center is boardered by chlorite, leucoxene, sulfides. XRD data: $\mathrm{Mg}-\mathrm{Fe}$ chlorite with rare hydrated interlayers; quartz as trace.

473.17 Gabbro-dolerite medium-grained with gabbro-ophytic texture. Plagioclase (0.3-1 mm) - labrador ( An $\left._{62}\right)$. Clinopyroxene (augite) up to $0.5 \mathrm{~mm}$ in diameter.

Alteration is moderate. About $10 \%$ of rock is chloritized. Chlorite and leucoxene replace glass in groundmass.

$\mathrm{XRD}$ data: Fe-chlorite with rare hydrated interlayers; quartz as trace.

482.10 Dolerite blastoporphyritic fine-grained with ophytic texture. Rare euhedral sceleton sulfide crystals (up to $3 \mathrm{~mm}$ ). Groundmass: laths of plagioclase (up to $1 \mathrm{~mm}$ ) - $\mathrm{An}_{55-60}$; about $50 \%$ of rock is unhedral clinopyroxene (augite or Ti-augite), located in interstices between laths of plagioclase. Alteration is strong (about $50 \%$ of rock is altered). Pyroxene is partially uralitized and chloritized, earthy aggregates of leucoxene and grains of sphene occur in chloritized matrix; pyrite is also common.

XRD data: $\mathrm{Mg}$-Fe chlorite with rare hydrated interlayers, corrensite-like mineral (minor); quartz and amphibole as traces.

491.94 Pyroxene hyalopilitic basalt.

Alteration is moderate. Brown glass is replaced by chlorite, traces of zeolite.

XRD data: Fe-Mg chlorite with rare hydrated interlayers; quartz and amphibole as traces.

500.70 Congo-dolerite medium-grained with ophytic texture, Clinopyroxene (augite), plagioclase (labrador). Opaques: Ti-magnetite. Alteration is strong (about $50 \%$ of rock is altered). Pyroxene is replaced along cracks by chlorite and uralite. Patches about $1 \mathrm{~mm}$ in diameter are composed of fine-grained quartz, chlorite, leucoxene and in some cases epidote. Veinlets: epidote (in zolbandes), chlorite and chalcedony (in center). XRD data: $\mathrm{Mg}$-Fe chlorite with about $10 \%$ of hydrated interlayers; corrensite-like mineral (minor); quartz and amphibole as traces.

501.88 Congo-dolerite (the same as in Sample 62R-1, 70-72 cm). Opaques:Ti magnetite and sparse blastoporphyritic sulfide crystals (up to 2 mm). Alteration is strong (about $50 \%$ of the rock is altered by chloritizationand sulfidization). Leucoxene replaces Ti-magnetite. Aggregates of quartz grains and sulfidization). Leucoxene replaces Ti-magnetite. Aggregates of quartz grains occur $(0.1 \mathrm{~mm})$. Plagioclase is partially replaced by albite. XRD data: Mg-Fe chlorite with about $10 \%$ of hydrated interlayers; corrensite-like mineral (minor); amphibole as trace.

510.20 Congo-dolerite (the same as in Sample 62R-1, 70-72 cm).

Alteration is strong (about $25 \%$ of rock is chloritized). Chlorite and epidote replace clinopyroxene. Microcracks are infilled by chlorite and epidote. Hydrogamet, leucoxene, sphene were found in chlorite pseudomorphs alter pyroxene. XRD data: Fe chlorite; amphibole as trace.

519.58 Dolerite medium-grained with ophytic texture

Alteration is moderate (about $25 \%$ of rock is chloritized). Chloriter eplaces clinopyroxene, plagioclase and glass. Uralite and chlorite replace amphibole.

XRD data: Mg-Fe chlorite; amphibole as trace.

560.19 Basalt ophyric, with vitrophyric texture.

Alteration is moderate. groudmass glass is chloritized.

XRD data: Fe-chlorite; quartz and amphibole as traces.

601.63 Plagioclase dolerite porhyric with ophytic texture. Phenocrysts: plagioclase (3-5 mm) up to 20\%; clinopyroxene (0.5-2 mm); olivine. Some plagioclase grains are sharply zoned: with bitovnite in center; labrador $\left(\mathrm{An}_{65}\right)$ - in periphery. Opaques: Ti-magnetite, sphene. Alteration is moderate (about 10\% of rock is chloritized). Pyroxene and plagioclase are replaced by chlorite and leucoxene. Pseudomorphs of chlorite and leucoxene replacing pyroxene and olivine. Epidote and albite also occur. $\mathrm{XRD}$ data: $\mathrm{Mg}$-Fe-chlorite; quartz as trace.

610.93 Olivine-plagioclase basalt porphyritic with microlitic texture. Groundmass: microlites of plagioclase and pyroxene, glass Alteration is moderate. Chlorite replacing olivine, pyroxene, and glass. There are chlorite and aggregates of small quartz grains, sulfides in interstices. XRD data: Fe chlorite; quartz and amphibole as trace.

647.35 Plagioclase basalt with pilotaxitic texture. Plagioclase-labrador $\left(\mathrm{An}_{56}\right)$ is about $20 \%$. Groundmass: microlites of plagioclase, volcanic glass with disseminated opaque mineral (magnetite?).

Alteration is moderate (chloritization). There are quartz, albite, andsulfides (in center) in veinlets.

XRD data: Mg-Fe chlorite with rare hydrated interlayers.

686.96 Dolerite fine-grained aphyric with doleritic texture. Plagioclase-labrador $\left(\mathrm{An}_{55}\right) 0.5-0.7 \mathrm{~mm}$, clinopyroxene-augite $(0.1-0.2 \mathrm{~mm})$. Opaques: Timagnetite, leucoxene.

Alteration is low (chloritization is about $5 \%$ of rock). Chlorite replaces groundmass glass and pyroxene.

XRD data: Fe chlorite with rare hydrated interlayers; quartz as trace.

687.47 Pyroxene basalt with intersertal texture.Alteration is moderate. Chloritization of glass and pyroxene. XRD data: Fe chlorite with rare hydrated interlayers; quartz and amphibole as traces.

734.35 Dolerite fine-grained aphyric amygdoloidal with ophytic texture.Clinopyroxene ( $50 \%$ ), plagioclase ( $50 \%$ ). Alteration is strong (about $30 \%$ of rock is chloritized). Plagioclase is replaced by albite, chlorite and epidote. Pyroxene is replaced by chlorite. Earthen aggregates of leucoxene and small grains of sphene replacing pyroxene. Amygdules (1-3 mm, about $40 \%$ of rock) are filled by aggregate of chlorite (clinochlore). In the central part of some amygdules there is epidote.

737.56 Olivine-pyroxene basalt amygdoloadal. Opaques: pyrite, Ti-magnetite. Alteration is strong (about $30 \%$ of rock is chloritized). There are chlorite and epidote in amygdules. Zoiside also presents. Pyroxene is partially replaced by uralite. Ti-magnetite is partially replaced by sphene.

XRD data: Fe chlorite with rare hydrated interlayers; quartz and amphibole as traces. 
Table 4 (continued).

\begin{tabular}{|c|c|c|}
\hline $\begin{array}{l}\text { Core, section } \\
\text { interval }(\mathrm{cm})\end{array}$ & $\begin{array}{l}\text { Depth } \\
\text { (mbsf) }\end{array}$ & Description \\
\hline $18 \mathrm{R}-2,84-85$ & 745,86 & $\begin{array}{l}\text { Gabbro-dolerite coarse-grained with poikilophyric texture. Clinopyroxene (up to } 3 \mathrm{~mm} \text { ) with inclusions of plagioclase (labrador-An } \mathrm{n}_{65} \text { ). In interstices } \\
\text { among laths of plagioclase there are sometimes small grains of orthoclase with abundant needle-shaped apatite and with grains of leucoxene,epidote, } \\
\text { uralite. } \\
\text { Alteration is strong. Chlorite and uralite replace pyroxene. Leucoxene, epidote, sulfides. } \\
\text { XRD data: Fe chlorite; amphibole as trace. }\end{array}$ \\
\hline $20 \mathrm{R}-1,64-66$ & 763.14 & $\begin{array}{l}\text { Gabbro-dolerite (same as in Sample } 18 \mathrm{R}-2,84-85 \mathrm{~cm} \text { ). } \\
\text { Alteration is strong (about } 30 \% \text { of rock is chloritized). Orthopyroxene is replaced by chlorite and uralite. Chlorite infilling numerous microcracks. } \\
\text { Sulfides. } \\
\text { XRD data: Fe-Mg chlorite with about } 10 \% \text { of hydrated interlayers, corrensite-like mineral (minor); quartz and amphibole as traces. }\end{array}$ \\
\hline $23 \mathrm{R}-1,57-59$ & 792.07 & $\begin{array}{l}\text { Dolerite medium-grained blastoporphyric with ophitic texture. Plagioclase } 0.5-1 \mathrm{~mm} \text { (labrador } \mathrm{An}_{55} \text { ) about } 50 \% \text {. Clinopyroxene (augite or Ti-augite) } \\
\text { about } 50 \% \text {. Opaques: sphene, leucoxene, Ti-magnetite, sulfides. } \\
\text { Alteration is moderate. Chlorite replacing pyroxene and glass in groundmass, infilling pores and cracks. Sphene and Ti-augite are replaced by } \\
\text { leucoxene. Sulfides infilling pores and cracks. } \\
\text { XRD data: Fe chlorite; quartz and amphibole as trace. }\end{array}$ \\
\hline $25 \mathrm{R}-1,73-75$ & 811.53 & $\begin{array}{l}\text { Dolerite (same as in Sample } 23 \mathrm{R}-1,57-59 \mathrm{~cm} \text { ). } \\
\text { Alteration is moderate (about } 20 \% \text { of rock is chloritized). Clinopyroxene is replaced by chlorite. Plagioclase is replaced by chlorite, epidote, albite. } \\
\text { Sphene and Ti-augite are replaced by leucoxene. } \\
\text { XRD data: Fe chlorite with rare hydrated interlayers; quartz and amphibole as traces. }\end{array}$ \\
\hline $33 \mathrm{R}-1,81-83$ & 888.61 & $\begin{array}{l}\text { Dolerite (same as in Sample } 23 R-1,57-59 \mathrm{~cm} \text { ). } \\
\text { Alteration is moderate (about } 25 \% \text { of rock is chloritized). } \\
\text { XRD data: Fe chlorite; quartz and amphibole as trace. }\end{array}$ \\
\hline $36 \mathrm{R}-1,3-5$ & 916.93 & $\begin{array}{l}\text { Microdolerite aphyric with microdoleritic texture. Plagioclase } 0.1-1 \mathrm{~mm} \text { (labrador } \mathrm{An}_{52-55} \text { ). Clinopyroxene } 0.1-0.3 \mathrm{~mm} \text {. } \\
\text { Alteration is moderate (about } 30 \% \text { of rock is chloritized). Plagioclase and pyroxene are chloritized and leucoxenized. Plagioclase is replaced by albite } \\
\text { and zeolite. } \\
\text { XRD data: Fe chlorite; quartz as trace. }\end{array}$ \\
\hline $\begin{array}{l}\text { 139-858F- } \\
\text { 26R-1,93-95 }\end{array}$ & 259.53 & $\begin{array}{l}\text { Olivine-pyroxene basalt porhyric, with hyalopilitic, partially with vitro-phyric texture, vesicular. } \\
\text { Alteration is low. Olivine, pyroxene and plagioclase are partially replaced by chlorite. Chlorite infilling pores ( } 0.3-1.5 \mathrm{~mm} \text { in diameter) and cracks. } \\
\text { XRD data: Fe-Mg chlorite with about } 10 \% \text { of hydrated interlayers corrensite-like mineral; quartz as trace. }\end{array}$ \\
\hline $27 \mathrm{R}-1,25-27$ & 268.05 & $\begin{array}{l}\text { Basalt (same as in Sample 26R-1, 93-95 cm). } \\
\text { Alteration is low (same as in Sample } 26 \mathrm{R}-1,93-95 \mathrm{~cm} \text { ). } \\
\text { XRD data: Fe-Mg chlorite with about } 10 \% \text { of hydrated interlayers; corrensite-like mineral; quartz and amphibole as trace. }\end{array}$ \\
\hline $28 \mathrm{R}-1,43-45$ & 277.93 & $\begin{array}{l}\text { Olivine-plagioclase basalt porphyric, with hyalopilitic texture. } \\
\text { Alteration is low. Chloritization of phenocrysts and groundmass. } \\
\text { XRD data: } \mathrm{Fe}-\mathrm{Mg} \text {-chlorite with about } 10 \% \text { hydrated interlayers; corrensite-like mineral. }\end{array}$ \\
\hline $\begin{array}{l}\text { 139-858G- } \\
\quad \text { IR-1, 14-16 }\end{array}$ & 276.94 & $\begin{array}{l}\text { Olivine-plagioclase basalt slightly porphyric with microdoleritic texture. } \\
\text { Alteration is moderate. Chlorite replaces glass, partially replaces plagioclase and pyroxene; olivine is totally altered. Albite replace plagioclase. } \\
\text { XRD data: Fe-Mg chlorite with about } 10 \% \text { of hydrated interlayers;corrensite-like mineral (minor). }\end{array}$ \\
\hline $4 \mathrm{R}-1,35-37$ & 306.15 & $\begin{array}{l}\text { Olivine-plagioclase basalt with hyalopilitic-porphyric texture. Opaques:rare sulfides. } \\
\text { Alteration is moderate. Phenocrysts and glass in groundmass are chloritized. } \\
\text { XRD data: Fe-Mg chlorite with about } 10 \% \text { of hydrated interlayers; corrensite-like mineral. }\end{array}$ \\
\hline 7R-1, 32-34 & 335.12 & $\begin{array}{l}\text { Basalt (same as in Sample } 4 \mathrm{R}-1,35-37 \mathrm{~cm} \text { ). } \\
\text { Alteration is moderate. Phenocrysts and glass in groundmass are chloritized. } \\
\text { XRD data: Mg-Fe chlorite with about } 10 \% \text { of hydrated interlayers; corrensite-like mineral (minor). }\end{array}$ \\
\hline $14 \mathrm{R}-1,10-12$ & 403.70 & $\begin{array}{l}\text { Plagioclase basalt porphyric amygdoloidal with microdoleritic texture. } \\
\text { Alteration is moderate (about } 15 \% \text { of rock is chloritized). Chlorite replacing pyroxene and infilling amygdules. Opal, chalcedony, and albite partially } \\
\text { replace plagioclase. } \\
\text { XRD data: Mg-Fe chlorite with rare hydrated interlayers; quartz and amphibole as trace. }\end{array}$ \\
\hline $16 \mathrm{R}-1,75-77$ & 423.65 & $\begin{array}{l}\text { Dolerite medium-grained amygdules with ophyric partialy poicilophitic texture. Lathes of plagioclase } 0.5-1 \mathrm{~mm} \text {, clinopyroxene. } \\
\text { Alteration is strong (about } 50 \% \text { of rock is chloritized). Chlorite and leucoxene replace pyroxene. Albite and epidote replacing plagioclase. Amygdules } \\
(0.5-4 \mathrm{~mm}) \text { are infilled by chlorite, in other cases - by chlorite, epidote and quartz. Glass in groundmass is replaced by chlorite and epidote. } \\
\text { XRD data: Fe chlorite; quartz and amphibole as trace. }\end{array}$ \\
\hline
\end{tabular}

to $25 \%$ carbonate. The carbonates include biogenic calcite (strongly recrystallized foraminifers and nannofossils), diagenetic calcite concretions, microconcretions, and burrow-fills. Dolomite was found in Core $139-858 \mathrm{C}-2 \mathrm{H}$ by shipboard XRD. The biogenic carbonate predominates in the coarse fraction (Table 3), constituting $85 \%$ to $91 \%$ in the light fraction. Bulk XRD data show somewhat lower quartz and feldspar contents than in the sediments below (Table 2); quartz prevails over feldspars. Pyrite, which is common in smear slides, is "rare."

The coarse heavy-mineral fraction (Table 3 ), however, is strongly dominated by pyrite $(84 \%-86 \%)$ that is partially oxidized. The rest is composed of iron oxide minerals, hornblende, epidote, apatite, and possibly authigenic barite. Quartz and feldspar occur in almost equal quantities in the light fraction, which is dominated by biogenic and partially authigenic carbonates. We found a zeolite (weak reflections of clinoptilolite(?) are recorded by XRD analyses). The clay fraction (Table 1, Fig. 8) is composed of Fe chlorite, hydromica, and smectite. The latter may be authigenic, precipitated from the hydrothermally affected ocean bottom waters.

Zone II ( 20 to about $54 \mathrm{mbsf}$ ) coincides with lithologic Subunit IIIB and contains among authigenic minerals quartz, pyrite, chlorite, and anhydrite. It is highly variable in mineralogy, demonstrating rather strong hydrothermal effects. The sediments are well indurated in this zone, especially in the coarse-grained turbiditic interbeds, which are cemented by carbonate and/or by gypsum and anhydrite. Pyrite also serves as a cement and authigenic $\mathrm{Mg}$-Fe chlorite is likely an important factor causing induration of the clay. 


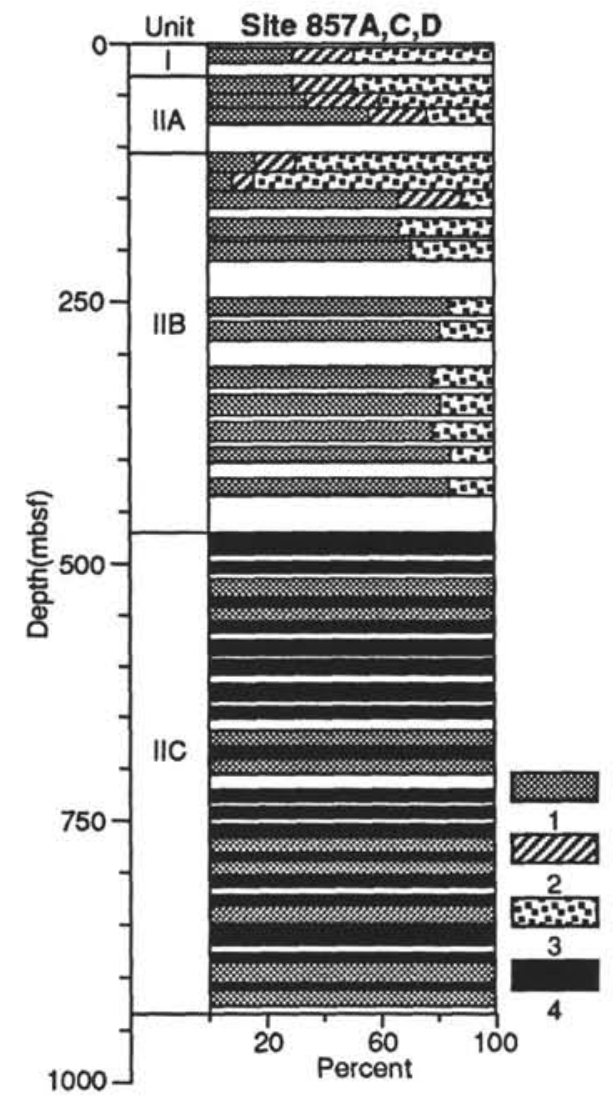

Figure 7. Variations in clay mineral composition, Site $857.1=$ chlorite; $2=$ mica; 3 = smectite; 4 = basalt.

Zone III (54-91.6 mbsf) coincides with lithostratigraphic Subunit IIC. It is characterized by abundant quartz, high chlorite and pyrite, and low mica and epidote. Sulfates and carbonates are absent.

\section{Hole $858 D$}

The degree of hydrothermal alteration in Hole $858 \mathrm{D}$ increases downhole as one secondary mineral association replaces another. Although gradual, these changes in mineralogy define three zones.

Zone I ( 0 to about $18 \mathrm{mbsf}$ ) is represented by nonlithified hemipelagic silty clay rather weakly affected by hydrothermal alteration. The zone coincides with lithostratigraphic Unit I and is similar to the upper zones in other holes at this site. The calcite content is rather high in samples from Core 139-858D-1H-1, but decreases to trace amounts in Cores $1 \mathrm{H}-2$ through $2 \mathrm{H}-3$. Dolomite was identified in Core $2 \mathrm{H}$. Epidote, anatase, pyrite and barite were detected in trace amounts. The clay fraction (Table 1, Fig. 9) is dominated by smectite (above 60\%) with minor Mg-Fe chlorite (about 30\%) and low mica content.

Zone II (18 to approximately $25 \mathrm{mbsf}$ ) is a well-developed zone of sulfate precipitation, as indicated by high anhydrite and gypsum contents. Pyrite also occurs on XRD records, as well as traces of epidoteclinozoisite, ankerite (?) and titanium-bearing minerals (Table 2). The zone is characterized by increased quartzification, indicated by high quartz content and decrease in feldspars.

Transition to Zone III at about 25-28 mbsf is marked by disappearance of feldspars and sulfates and a decrease in mica content. Epidoteclinozoisite, rutile, anatase, and barite were detected in thin sections and smear slides. Pyrite is present. Quartzification is apparent.

Zone III ( $25-28$ to $40.7 \mathrm{mbsf}$ ) coincides with lithologic Subunit IID. A quartz-wairakite vein was detected from a metamorphosed siltstone. The vein is composed of euhedral wairakite and quartz with minor sphalerite. According to shipboard study such veins are com-
Table 5. Microprobe analyses of secondary minerals in igneous rocks, Leg 139 .

\begin{tabular}{lrrrr}
\hline Component & \multicolumn{1}{c}{1} & \multicolumn{1}{c}{2} & \multicolumn{1}{c}{3} & \multicolumn{1}{c}{4} \\
\hline $\mathrm{SiO}_{2}$ & 55.81 & 28.40 & 70.60 & 30.74 \\
$\mathrm{TiO}_{2}$ & 0.01 & 0.00 & 0.01 & 0.00 \\
$\mathrm{Al}_{2} \mathrm{O}_{3}$ & 0.11 & 18.42 & 21.05 & 17.96 \\
$\mathrm{Cr}_{2} \mathrm{O}_{3}$ & 0.32 & 0.00 & 0.00 & 0.22 \\
$\mathrm{FeO}$ & 7.82 & 26.83 & 0.00 & 22.34 \\
$\mathrm{MnO}$ & 0.00 & 0.33 & 0.00 & 0.27 \\
$\mathrm{MgO}$ & 28.00 & 17.16 & 0.00 & 19.78 \\
$\mathrm{CaO}$ & 0.11 & 0.16 & 1.18 & 0.18 \\
$\mathrm{Na} \mathrm{O}_{2} \mathrm{O}$ & 0.00 & 0.13 & 9.35 & 0.00 \\
$\mathrm{~K}_{2} \mathrm{O}$ & 0.00 & 0.00 & 0.00 & 0.00 \\
$\mathrm{Total}^{2}$ & 92.18 & 91.56 & 101.60 & 91.50 \\
\hline
\end{tabular}

Note: $1=$ talc replacing olivine (Sample $856 \mathrm{~A}-14 \mathrm{X}-1,34-38$ $\mathrm{cm}$ ); 2 = chlorite replacing pyroxene (Sample 857D-36R-1, $3-5 \mathrm{~cm}$ ); 3 = albite replacing plagioclase (Sample 858GIR-1, 14-16 cm); 4 = chlorite (Sample 858G-1R-1, 14-16 $\mathrm{cm})$.

mon in the lowermost part of the section. In thin sections we found also small segregations of a finely crystalline titanium-bearing mineral (anatase?) that may be authigenic.

Zone I is rather weakly affected by hydrothermal activity. Zone II is characterized by an increase in silica due to quartzification, indicated by high quartz content and by decrease in feldspars, and abundant precipitation of sulfate. Pyrite, epidote, ankerite (?), and titaniumbearing minerals are detected as traces. Zone III is marked by disappearance of feldspars and sulfates, decrease in mica, and appearance of analcime and wairakite. Chlorite, epidote, rutile, anatase, barite, and pyrite are present in trace amounts. Quartzification is apparent.

\section{Holes $858 F$ and $G$}

Basalt samples from Holes $858 \mathrm{~F}$ and $858 \mathrm{G}$ are strongly altered, mainly to chlorite (Table 4 ). The chlorite replaces primary minerals both in phenocrysts and in the groundmass and infills pores, cracks, and amygdules. In the basalt sequence at Site 858 the Fe content of the secondary chlorite decreases upward, from Fe chlorite below (Table 5) to a high-Mg corrensite-like mineral above (i.e., the same trend as described for Hole 857C). Albitization of plagioclase was detected in Hole $858 \mathrm{G}$ (Tables 5). Plagioclase has also been replaced by opal and chalcedony. In the lowermost sample studied, plagioclase is replaced by albite and epidote, and pyroxene is replaced by chlorite and leucoxene. Amygdules are infilled by chlorite, epidote, and quartz. Groundmass glass is chloritized and epidotized. XRD data show presence of quartz and amphibole in trace amounts.

\section{CORRELATION AND PROCESSES}

Sites 856,857 , and 858 can be arranged according to the degree and character of hydrothermal alteration of the sedimentary sequences recovered. Site 856 is least affected, especially Hole 856A. The composite section drilled at Site 857 seems to be intermediate, as the alteration zones are expanded over greater thickness and occur at greater depths. Site 858 shows the most extensive alteration at shallowest depths, with "condensed" hydrothermal zones within the shortest intervals. However, the mode of hydrothermal processes and the zonation seem to be almost the same throughout the area of Middle Valley drilled. The sites differ from each other by degree and intensity of the hydrothermal alteration, but not by the main trends of mineralogical changes of the primary terrigeneous sediments. Moreover, the basalts from the sills are altered in the same way as the surrounding and intercalated sediments.

\section{Zone III}

Zone III is present in all holes except Hole 856A. The zone is characterized by strong hydrothermal alteration of sediments and 


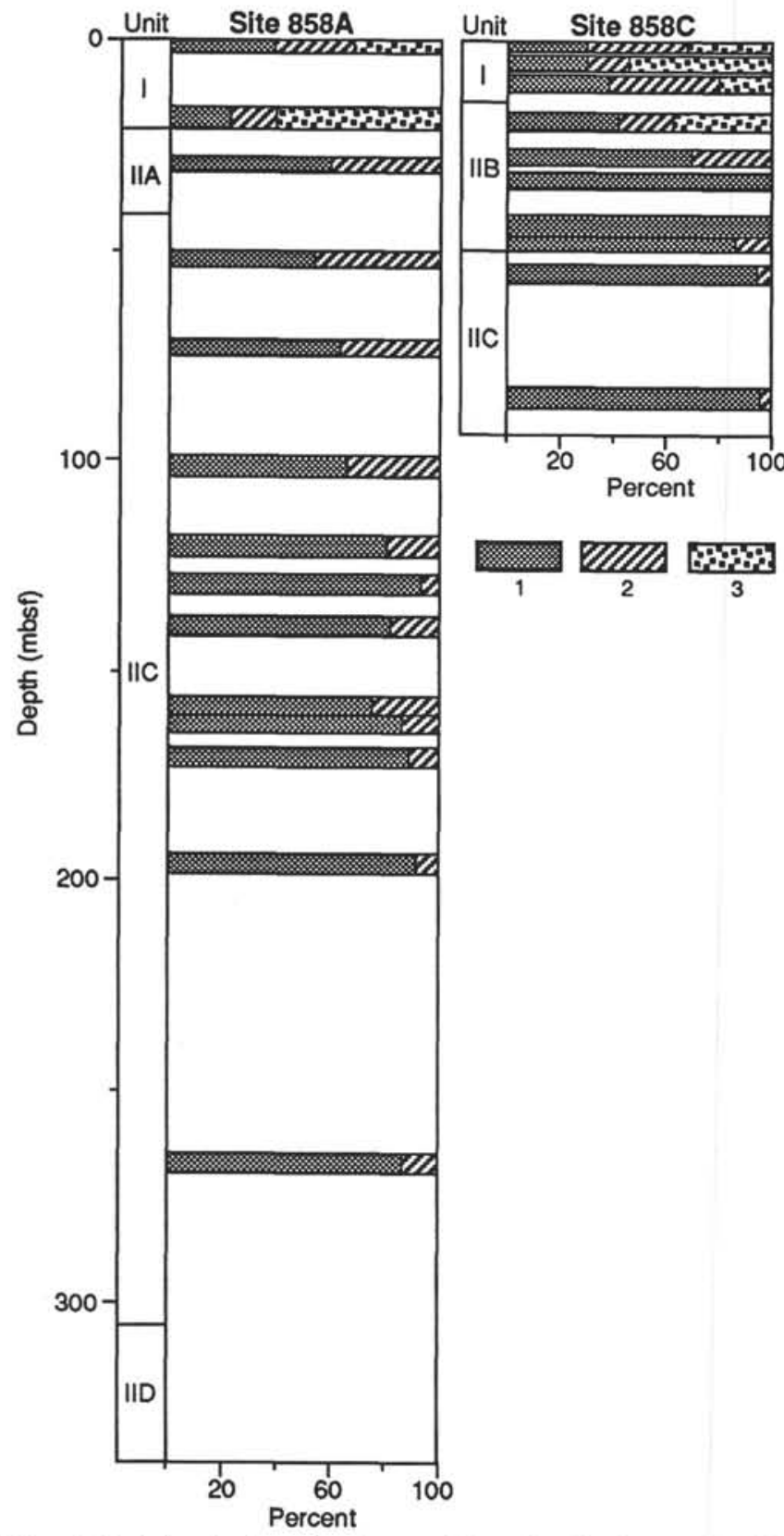

Figure 8. Variations in clay mineral composition of the clay fraction, Site 858, Holes $858 \mathrm{~A}$ and $858 \mathrm{C} .1=$ chlorite $2=$ mica; $3=$ smectite.

basalts. The main hydrothermal minerals are pyrite, chlorite, and quartz. At Site 857 epidote is also present. Wairakite and analcime appear in sediments from Holes 858A and 858D.

Quartzification (silicification) is best developed in this zone. Quartz formation requires significant silica input from hydrothermal fluids. The extensive silicification leads to destruction (up to total disappearance) of feldspar, as occurs in the lower zones in Hole 858D. Potassium is leached from the quartz-rich zones as mica and $\mathrm{K}$-feldspar is altered. Calcium is commonly removed or is partially tied up in epidote and wairakite. Chloritization is one of the most important alteration processes in Zone III. Fe-chlorite was detected only in the extensively altered lower part of the sediment sequence. Chloritization is accompanied by destruction of the detrital mica/hydromica with removal of potassium. Feldspars are also affected by the chloritization, as is hornblende. Epidotization is best manifested in the lower part of the composite section at Site 857, where the chlorite-epidote-quartz

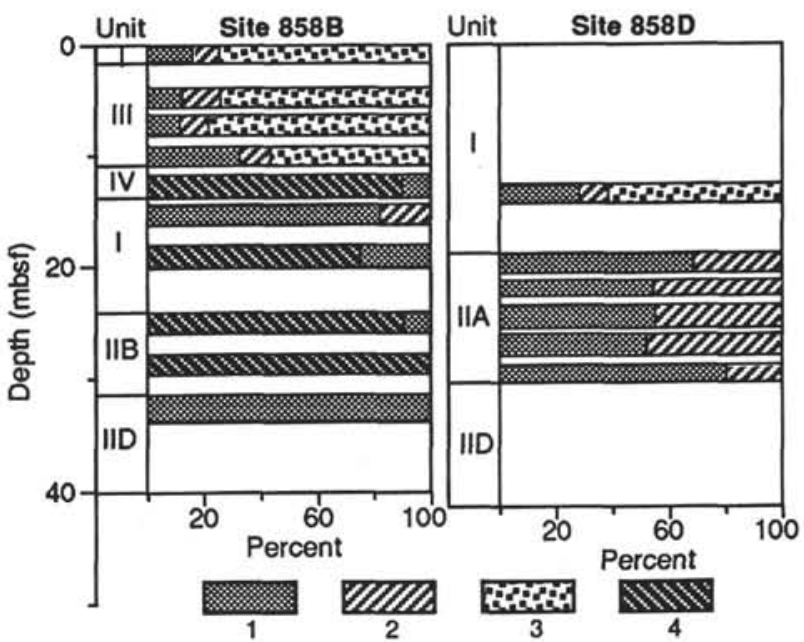

Figure 9. Variations in clay mineral composition of the clay fraction, Site 858, Holes $858 \mathrm{~B}$ and $858 \mathrm{D} .1=$ chlorite; $2=$ mica; $3=$ smectite; $4=$ corrensitelike mineral.

metamorphic rocks are intercalated with numerous doleritic sills. A hydrothermal origin of epidote is indicated by structural interrelations of minerals in thin section and by a euhedral habit. Hydrothermal epidote is also present in strongly altered and silicified sandstones from other holes. Sulfide precipitation is apparently caused by extensive influx of sulfur with upwardly moving hydrothermal fluids.

Titanium-bearing minerals-rutile, anatase, sphene, and leucoxene-occur in different alteration zones, but their hydrothermal origin is best manifested in the highly silicified zones. In such zones, euhedral crystals of titanium-bearing minerals form clusters in pores and interstices. In some cases, the fine-grained mineral aggregates cement clastic grains.

Many of the minerals found in the altered sediments were also observed among the alteration products of basalts and dolerites in sills (Table 4). Chloritization is the main alteration process, with chlorite replacing all of the primary volcanic minerals-plagioclase, pyroxene, and olivine, as well as groundmass glass. Chlorite also infills amygdules and cracks. The alteration of basalts occurred under greenschist facies conditions, as has been well studied in volcanic rocks dredged from the mid-oceanic rifts and transform faults (e.g., Melson and Van Andel, 1966; Murdmaa and Prokoptsev, 1968; Cann, 1969). The parageneses recognized in Leg 139 rock samples are similar to those in altered sediments between and below the doleritic sills, as described above. The alteration requires input of sodium and silica (for albitization and quartzification) along with water necessary for hydration, and removal of $\mathrm{Ca}$.

Previous study of mid-oceanic ridge basalts from DSDP/ODP sites (Kurnosov, 1986) identified the smectite facies of metamorphism within the upper layer of oceanic crust. The evidence for greenschist facies (mainly chloritization) was observed at much greater depths (about 600 to $1000 \mathrm{~m}$ deep within the basaltic layer of the oceanic crust), although greenschist facies rocks have been recovered in numerous dredge hauls from the mid-oceanic spreading centers, including metamorphosed gabbro, diabase, and basalt (Murdmaa and Prokoptsev, 1968). The Leg 139 results, however, indicate that within extremely active high-temperature hydrothermal fields such as those in Middle Valley, the greenschist facies may form only a few tens of meters below the seafloor (Site 858). The mineral parageneses both in basalts and in sediments indicate the greenschist facies.

Formation of mineral assemblages with mainly chlorite and quartz is estimated at temperatures between $350-450^{\circ} \mathrm{C}$ (Marakushev, 1968). Experimental data show that wairakite forms at $300^{\circ}-350^{\circ} \mathrm{C}$ (Kholodkevich et al., 1981). Alteration processes in Zone III indicate "nonoxidative" conditions with circulation of fluids rich in $\mathrm{Si}, \mathrm{Ca}$, and $\mathrm{Fe}$. 


\section{Zone II}

Zone II was observed in all holes. The main hydrothermal minerals for the zone are pyrite, chlorite, and/or a corrensite-like mineral. Anhydrite, quartz, and carbonate are present, and epidote was found in sediments recovered at Site 857.

Sulfide precipitation as best exhibited by formation of semimassive sulfide recovered in Holes $856 \mathrm{~B}$ and $858 \mathrm{~B}$ is dominated by pyrite and pyrrhotite. Formation of these minerals requires considerable amounts of free iron to tie up the sulfur. Otherwise the latter migrates with circulating interstitial water as dissolved $\mathrm{H}_{2} \mathrm{~S}$. When oxygenated conditions are encountered, $\mathrm{H}_{2} \mathrm{~S}$ is oxidized to sulfate, which reacts with $\mathrm{Ba}^{2+}$ and $\mathrm{Ca}^{2+}$ ions to form barite and anhydrite. Anhydrite can subsequently be hydrated and replaced by gypsum at lower temperatures. The limiting role of Fe availability was suggested for early diagenetic sulfide formation (Volkov, 1984), but the mechanism perhaps works also in the hydrothermal sulfur system.

The complicated interplay between redox processes, iron and sulfur input from hydrothermal fluids, and the thermodynamic potential of $\mathrm{H}_{2} \mathrm{O}$ as a factor of hydration-dehydration (Marakushev, 1968) leads to complicated interrelations between the sulfates and sulfides precipitated from the fluids into sediment interstices and fissures. The processes are apparently multiple, repeatedly alternating with each other in the hydrothermal system active today. Experimental data show that anhydrite forms at $200^{\circ}-300^{\circ} \mathrm{C}$ (Hajash and Archer, 1980; Seyfried and Bischoff, 1981).

The corrensite-like mineral (mixed-layer smectite/chlorite) we interpret as a metastable phase that is a precursor to chloritization. It forms during active and rapid hydrothermal alteration under relatively high temperature conditions within the active vent area (Hole 858B), where it constitutes almost the total clay mineral content in a zone between the smectite-bearing zone above and the chlorite zone below. This assumption is consistent with the thermodynamics of metamorphic reactions in the system $\mathrm{MgO}-\mathrm{Al}_{2} \mathrm{O}_{3}-\mathrm{SiO}_{2}$ with excess water, which lead to the following progressive alteration parageneses with increasing temperature: Montmorillonite-Montmorillonite + chlorite (= corrensite $?)-$ Montmorillonite + chlorite + quartz - chlorite + quartz, at temperatures between $350^{\circ}-450^{\circ} \mathrm{C}$ (Marakushev, 1968). The temperatures are obtained experimentally for $2 \mathrm{Kbar}$ water pressure and should be diminished under lower fluid pressure conditions. High $\mathrm{Mg}$ activity is probably required for abundant corrensite formation.

Carbonates reflect decreasing temperature of hydrothermal fluids. Carbonates of hydrothermal (or of hydrothermally intensified diagenetic) origin are represented mainly by low-magnesium calcite, which occurs as concretions (described by Davis, Mottl, Fisher, et al. [1992] but not included in our samples), sparry cement, finely crystalline micritic matrix, and recrystallization of foraminiferal tests. Abundant calcite precipitation is confined to the uppermost alteration zone characterized by the lowest temperatures and increased alkalinity and $\mathrm{pH}$ values. The main source of the calcite constituents is likely dissolution and recrystallization of biogenic carbonates below and within the calcification zone. However, carbon isotopic analyses (Baker et al., this volume) show low negative values of $\delta^{13} \mathrm{C}$ for authigenic carbonate from the concretions, indicative of methane-derived $\mathrm{CO}_{2}$ as a source of carbon. This is consistent with our data obtained from a Kuril island arc submarine cold spring associated with methane hydrates (Zonenshain et al., 1987). Abundant diagenetic calcite/aragonite crusts and concretions were observed there both on the seafloor and within Holocene organic-rich volcaniclastic sediments. The $\delta^{13} \mathrm{C}$ measured in those authigenic carbonates is as low as $-49 \%$. We interpreted this value to be a result of bacterial methane oxidation, the methane being derived from a rather mature hydrocarbon source deep within the sediment sequence. In the case of Middle Valley carbonate concretions the source may be hydrothermally metamorphosed (maturated) organic carbon. Other carbonates found in trace amounts are dolomite, ankerite(?), and aragonite(?).

\section{Zone I}

Zone I was found in all holes except Hole 856B. The zone coincides with the lithologic Unit I. In Hole 856A the zone contains mainly clastic "background" sediment nearly free of smectites. At Sites 857 and 858 the zone contains authigenic smectites.

Smectite is most abundant in Hole $858 \mathrm{~B}$, drilled directly into the active vent area, where the upper unit is represented by metalliferous hemipelagic clay. The hydrothermally derived smectite is confined to the hemipelagic clay because this clay is accumulating continuously by "normal" sedimentation at a rate comparable to that of the hydrothermal input. The underlying turbidites as products of "catastrophic" sedimentation are derived from the nearby continental slope and are deposited instantaneously, having no time to incorporate a hydrothermal contribution. An alternative explanation, that smectite disappears at depths beneath the surface layer by hydrothermal alteration, is less probable, as the thermal stability of smectites extends to much higher temperatures than those suggested for the weakly altered upper zone.

\section{SUMMARY AND CONCLUSIONS}

The mineralogical data on the hydrothermally altered sediments from Sites 856,857 , and 858 generally confirm the preliminary conclusions made by shipboard scientists, but reveal additional findings.

Sites 856,857 , and 858 can be arranged according to the degree of hydrothermal alteration of the sedimentary sequences recovered. Site 856 is least affected, especially Hole $856 \mathrm{~A}$. The deep composite section drilled at Site 857 seems to be intermediate, as the alteration zones occur deeper and over greater thickness. Site 858 best shows the extensive alteration at shallowest depths, with the "condensed" hydrothermal zones within the shortest intervals. However, the mode of hydrothermal processes and the zonation seem to be almost the same throughout Middle Valley.

The newly formed hydrothermal minerals found in sediments include silicates-aluminosilicates (quartz, albite[?], chlorite, corrensite-like mineral, smectites, hydromica, epidote group, fibrous amphibole[?], garnet[?], wairakite, analcime, and talc), titaniumbearing minerals (rutile, anatase, sphene, and leucoxene), sulfates (anhydrite, gypsum, and barite), sulfides (pyrite and others not included in our study), and carbonates (calcite, dolomite, and aragonite[?]). The minerals occurring in abundant or common quantities are in bold; others are rare. The hydrothermal minerals listed have grown both in free space (cracks, pores, cavities, molds after dissolution of anhydrite, foraminiferal tests, empty burrows) and replacing primary detrital minerals. Both modes of crystallization occur when crystals (e.g., epidote) formed in interstitial spaces penetrate into the detrital grains. Recrystallization (regeneration) of quartz leading to quartz cementation is common in silicified sandstones. The primary detrital chlorite is perhaps also recrystallized during total chloritization of the sediments. Skeletal crystals and poikiloblasts of sulfides, anhydrite, gypsum, and sparry calcite occur as cementation of relatively fine-grained sediments, including silt and silty clay.

Along with crystallization of new mineral phases and replacement or recrystallization of primary minerals, the hydrothermal alteration includes partial or total destruction of the primary components. Among these processes are dissolution of biogenic carbonate and silica and destruction of detrital feldspars and mica. Dissolution of anhydrite leading to formation of rectangular molds is another such destructive process.

Three main hydrothermal zones are recognized in the sediment sequence at Sites 856,857 , and 858 . The lowermost Zone III with strong hydrothermal alteration is characterized by pyrite, chlorite, quartz, and epidote, wairakite, and analcime. The mineral assemblages both in altered sediments and in associated basalts were formed under greenschist metamorphic facies. Zone II with moderate hydrothermal alteration contains pyrite, chlorite, corrensite-like mineral, anhydrite, 
quartz, and carbonate. The uppermost Zone I contains mainly clastic sediments and authigenic smectites.

\section{ACKNOWLEDGMENTS}

We thank the reviewers for useful remarks. We are grateful to $\mathrm{O}$. Chudaev, E. Petrachenko, V. Vasilyeva, A. Sokolova, E. Pokrovskaya, and V. Sapin for help with analytical work and data processing.

\section{REFERENCES*}

Cann, J.R., 1969. Spilites from the Carlsberg Ridge, Indian Ocean. J. Petrol., 10:1-19.

Davis, E.E., Mottl, M.J., Fisher, A.T., et al., 1992. Proc. ODP, Init. Repts., 139: College Station, TX (Ocean Drilling Program).

Hajash, A., and Archer, P., 1980. Experimental seawater/basalt interaction: effects of cooling. Contrib. Mineral. Petrol., 75:1-13.

Kholodkevich, I.V., Kotov, N.V., and Kurnosov, V.B., 1981. Experimental study of the alteration of glassy volcanic rocks in distilled water and modelled seawater at high PT parameters. In Kossovskaya, A.G. (Ed.), Mineralogical Alteration of the Oceanic Rocks: Moscow (Science), 6772.

Kurnosov, V.B., 1986. Hydrothermal Alteration of Basalts in the Pacific Ocean and Metal-bearing Deposits, Using Data of Deep-sea Drilling: Moscow (Izd. Nauka).

Marakushev, A.A., 1968. Thermodynamics of the Metamorphic Hydration of Minerals: Moscow (Nauka).
Melson, W.G., and Van Andel, T.H., 1966. Metamorphism in the Mid-Atlantic ridge $22^{\circ} \mathrm{N}$ latitude. Mar. Geol., 4:234-246.

Murdmaa, I.O., and Prokoptsev, N.G., 1968. On the recovery of spilite in the Arabian-Indian Ridge rift zone. Dokl. Acad. Nauk, 181:458-461.

Rozanova, T.V., Driz, V.A., and Dmitrik, A.V., 1978. A hydrothermal pyroxene-amphibole asbestos rock from the Hess Deep. Litol. Polezn. Iskop., $3: 3-16$.

Seyfried, W.E., and Bischoff, J.L., 1981. Experimental seawater-basalt interaction at $300^{\circ} \mathrm{C}, 500$ bars, chemical exchange, secondary mineral formation and implications for the transport of heavy-metals. Geochim. Cosmochim. Acta, 45:135.

Sudo, T., Oinuma, K., and Kobayashi, K., 1969. Mineralogical problems concerning rapid clay mineral analysis of sedimentary rocks. Acta Univ. Carol., Geol. Suppl., 1:189-219.

Volkov, I.I., 1984. Geochemistry of Sulfur in the Ocean: Moscow (Nauka).

Zonenshain, L.P., Murdmaa, I.O., and Baranov, B.V., 1987. Submarine gasspring in the Sea of Okhotsk West from the Paramushir Island. Okeanologia (Moscow), 27:795-800.

- Abbreviations for names of organizations and publications in ODP reference lists follow the style given in Chemical Abstracts Service Source Index (published by American Chemical Society).

Date of initial receipt: 23 February 1993

Date of acceptance: 16 September 1993

Ms 139SR-267 


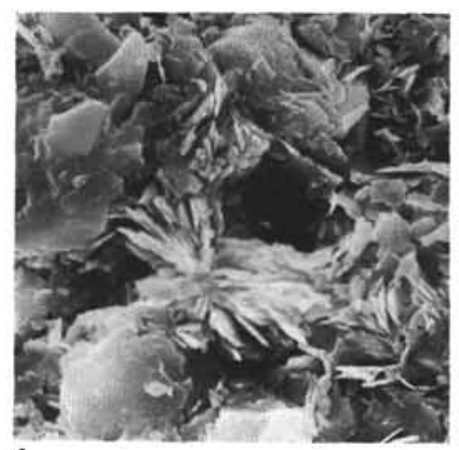

1

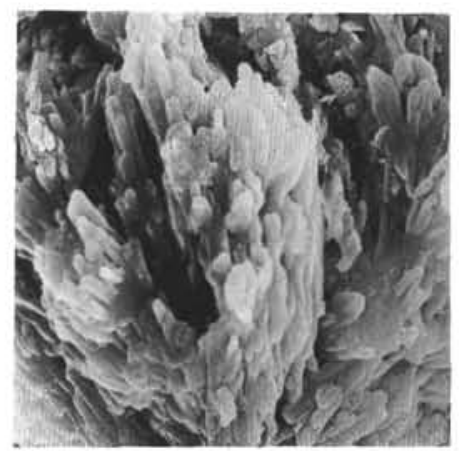

3

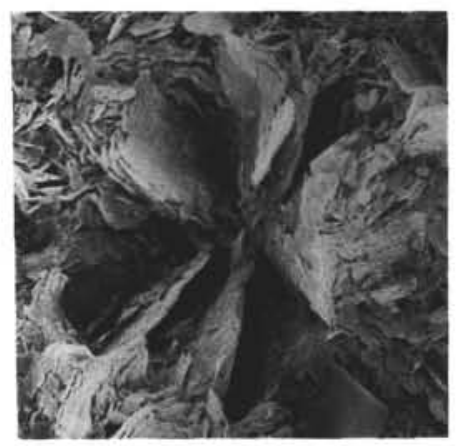

5

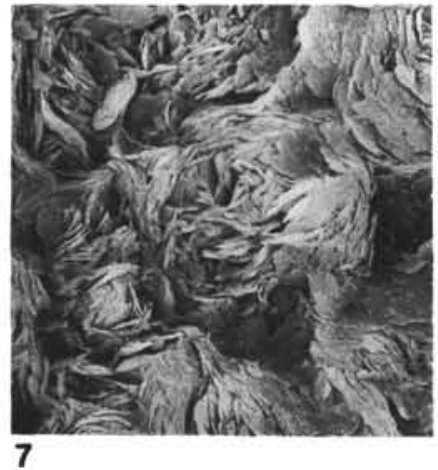

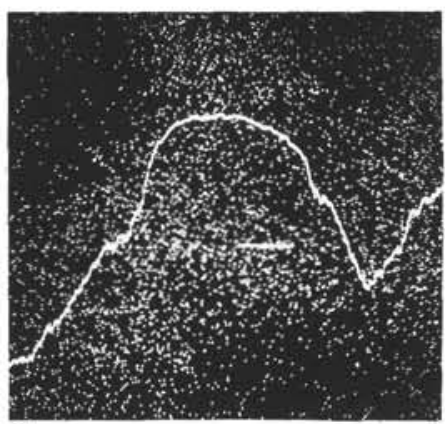

2
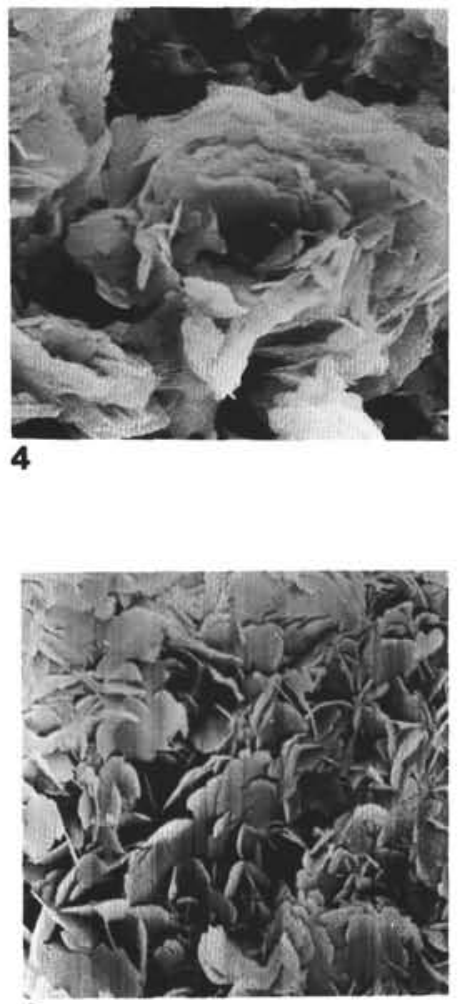

6

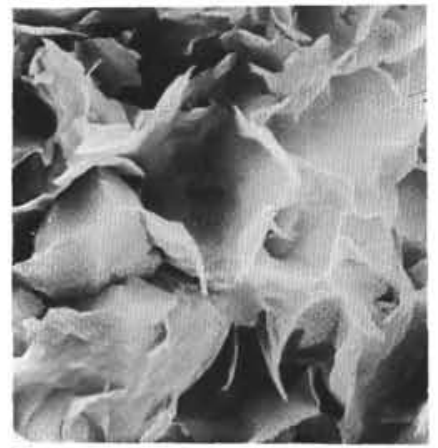

8

Plate 1. SEM micrographs of hydrothermal minerals from Leg 139 sediments: clay minerals. 1, 2. Radiated hydromica aggregate: Sample 139-856B-15X-4, 73-75 cm, $\times 2730$. (1) micrograph. (2) scanning microprobe measure of K. 3. Elongated platy crystals of hydromica in radiated aggregate: Sample 139-856A13X-4, 94-98 cm, $\times 2730$. 4-7. Different forms of chlorite: (4) rosette-like aggregate of curved flakes; Sample 139-856B-15X-3, 112-114 cm, $\times 2730$. (5) radiated aggregate; Sample 139-858A-39X-CC, 15-17 cm, $\times 1820$. (6) platy crystals; Sample 139-858A-21X-3, 19-22 cm, $\times 4550$. (7) cabbage-like spheroidal aggregates; Sample 139-858A-39X-CC, 15-17 cm, $\times 1820$. 8. Corrensite-like (mixed-layer) mineral; Sample 139-858B-5H-4, 14-18 cm, $\times 1820$. 


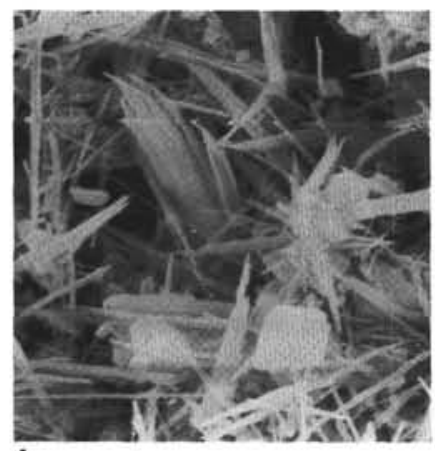

1

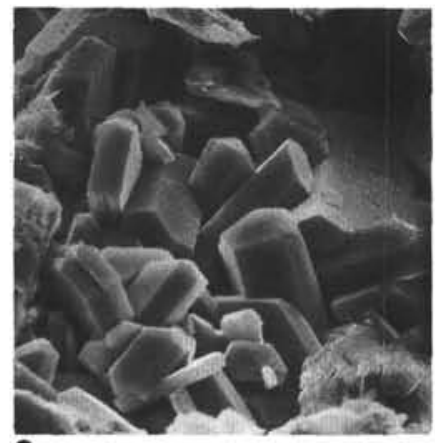

3
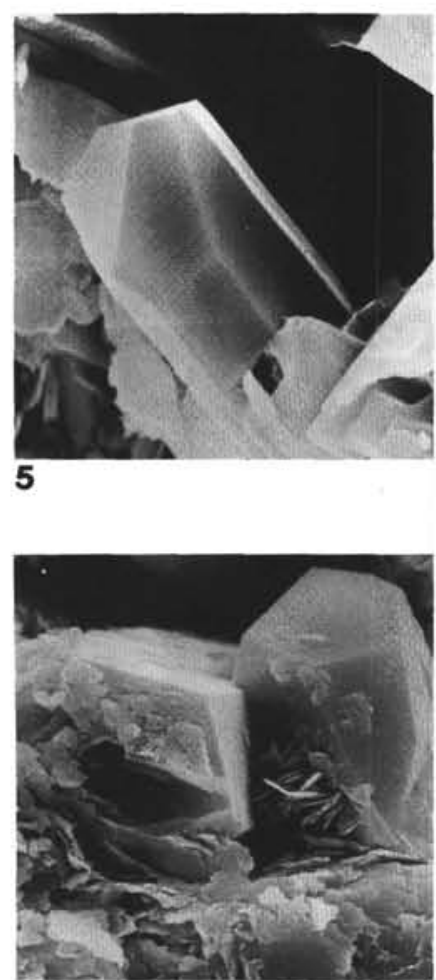

7

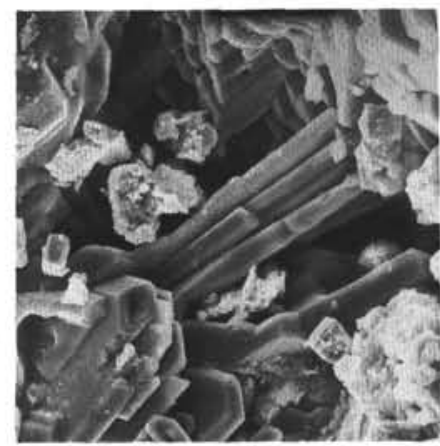

2

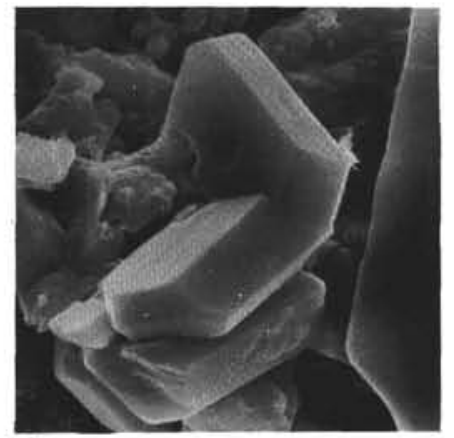

4

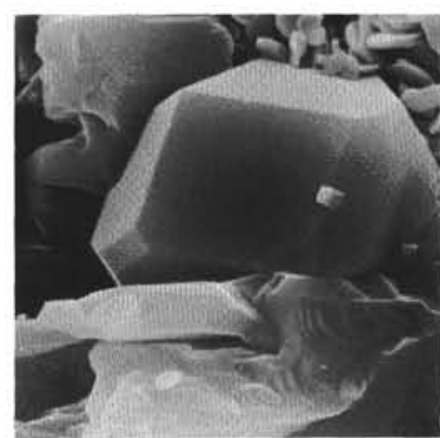

6

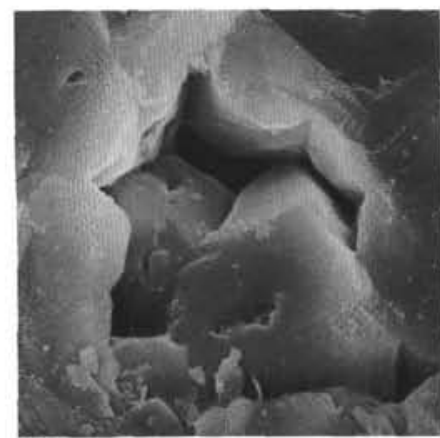

8

Plate 2. SEM micrographs of hydrothermal minerals from Leg 139 sediments: nonclay minerals. 1. Fibrous-diverse aggregate of amphibole (?) infilling recrystallized foraminiferal test; Sample 139-856B-2H-3, 106-110 cm, $\times 4550$. 2-4. Different habits of gypsum crystals. (2) Sample 139-858D-4H-4, 100-104 $\mathrm{cm}, \times 1820$. (3) Sample 139-858D-4H-4, 100-104 cm, $\times 910$. (4) Sample 139-858C-6H-3, 34-36 cm, $\times 2730$. 5-6. Euhedral epidote crystals growing in pore space: (5) Sample 139-857D-31R-1, 13-15 cm, $\times 4550$. (6) Sample 139-857D-37R-1, 42-43 cm, $\times 4550$. 7-8. Wairakite and analcime crystals in quartzwairakite-analcime vein in chloritized claystone matrix; Sample 139-858A-39X-CC, $15-17 \mathrm{~cm}, \times 3640$. 